$L B L--33186$

DE93 010452

\title{
Understanding Conformal Field Theory through Parafermions and Chern Simons Theory
}

\author{
Scott A. Hotes \\ Ph.D. Thesis \\ Department of Physics \\ University of California \\ and \\ Physics Division \\ Lawrence Berkeley Laboratory \\ University of California \\ Berkeley, CA 94720
}

November 1992

\section{WASTER}

This work was supported in part by the National Science Foundation under grant PHY90-21139 and by the Director, Office of Energy Research, Office of High Energy and Nuclear Physics, Division of High Energy Physics, of the U.S. Department of Energy under Contract No. DE-AC03-76́SFONo9s. 
November 19, 1992

LBL-33186

UCB-PTH-92/40

\title{
Understanding Conformal Field Theory through Parafermions and Chern Simons Theory *
}

\author{
Scott A. Hotes \\ Department of Physics \\ University of California \\ and \\ Theoretical Physics Group \\ Physics Division \\ Lawrence Berkeley Laboratory \\ 1 Cyclotron Road \\ Berkeley, California 94720
}

\begin{abstract}
Conformal field theories comprise a vast class of exactly solvable two dimensional quantum field theories. Conformal theories with an enlarged symmetry group, the current algebra symmetry, are a key ingredient to possible string compactification models. The following work explores a Lagrangian approach to these theories.

In the first part of this thesis, a large class of conformal theories, the so-called coset models, are derived semi-classically from a gauged version of the Wess-Zumino-Witten functional. A non-local field transformation
\end{abstract}

"This work was supported in part by the Director, Office of Energy Research, Office of High Energy and Nuclear Physics, Division of High Energy Physics of the U.S. Department of Energy under Contract DE-AC03-76SF00098 and in part by the National Science Foundation under grant PHY90-21139. 
to the parafermionic field description is employed in the quantization procedure. Classically, these parafermionic fields satisfy non-trivial Poisson brackets, providing insight into the fractional spin nature of the conformal theory. The W-algebra symmetry is shown to appear naturally in this approach.

In the second part of this thesis, the connection between the fusion algebra structure of Wess-Zumino-Witten models and the quantization of the Chern-Simons action on the torus is made explicit. The modular properties of the conformal model are also derived in this context, giving a natural demonstration of the Verlinde conjecture. The effects of background gauge fields and monopoles are also discussed. 


\section{Contents}

1 Introduction 1

I Parafermions and Conformal Field Theory 4

2 Lagrangian Models in Conformal Field Theories 4

3 Review of the Wess-Zumino-Witten Model 6

4 The Gauged Wess-Zumino-Witten Model 7

5 The Parafermionic Currents 9

6 The Poisson Bracket of the Parafermionic Currents 10

7 The Poisson Algebra and Multilocal Observables 15

8 The Classical W Algebras $\quad 19$

9 "Free Current" Realization of Parafermion Algebra 23

10 Quantization of the Parafermionic Current Algebra 25

II Chern-Simons Theory and Conformal Field Theory

$112 D$ Conformal Field Theory and Topological Field Theory 27

12 The Chern-Simons Action Functional 28

13 Quantization of the Chern-'imons Action 29

14 Modular Tranformations $\quad 34$ 
15 Fusion Rules and the Verlinde Conjecture

16 Background Gauge Fields and Monopoles

17 Three Necessary Calculations

39

18 Figures

44 


\section{Introduction}

The central mathematical apparatus employed in describing modern particle physics is the quantum field theory "QFT." As an axiomatic system, QFT is laden with ambiguities. Even in its linearized, or perturbative form, unitarily inequivalent canonical coordinates exist, renormalization procedures must be prescribed, and often the resulting series is only asymptotic. From this viewpoint, we have barely scratched the surface of the richness inherent in the full non-linear field theory consistently combined with the quantum theory.

The complexities of a full non-linear theory can be observed at the classicai level as well. A rele:vant example of such a theory is Einstein's theory of general relativity. Taken perturbatively, this theory correctly describes large scale phenomena such as the perihelion advance of Mercury, overcoming the shortcomings of Newton's theory of gravity. Taken as a full non-linear theory, however, exact solutions exist only for trivial systems, and very little is known concerning solutions to the Cauchy problem with non-trivial energy-momentum tensor.

In the last two decades, much attempt has been made at probing the inherent non-linear, or nonperturbative effects of a given QFT. In 1984, in the pioneering work of Belevin, et al. [14], a large class of non-trivial QFTs was shown to be completely solvable. These theories were a subclass of two-dimensional QFTs carrying a conformal symmetry. These theories were solvable in the sense that all field operators could be characterized, and in principle the correlators of these fields were finite and could be computed exactly. Thus began the age of conformal field theory "CFT."

Besides being an important class of solvable QFTs, conformal field theories arise naturally in string theory and the critical behavior of statistical systems. For this reason understanding the structure of CFTs and possibly classifying the space of rational CFTs (rationality is a technical point, implying a finite set of primary fields) has become a major endeavor of modern particle theorists.

An important class of conformal field theories, carrying an enlarged symmetry group, the current algebra symmetry, plays a key role in string compactification models. These conformal models have been shown by Witten [58] to be derived from a sigma model type Lagrangian, containing a topological WessZumino term. These are the Wess-Zumino-Witten (or WZW) models. Gauging 
the WZW action gives a wide class of conformal models, the so-called coset models. This follows a long history beginning with Witten's work on the skyrmion model [57].

The conformal symmetry group in two dimensions gives rise to an infinite dimensional algebra, the Virasoro algebra, admitting a non-trivial central element. Thus, the Hilbert space of a CFT can be defined by its decomposition under this algebra. The Hilbert space of a WZW model can further be decomposed under the action of the current algebra symmetry; this algebra is described as the semi-direct product of the Virasoro algebra with the corresponding affine Lie algebra. This characterization also provides a simple description of the operator product expansions of the field variables. Classifying the allowable fusion algebras by employing general consistency requirements is one intriguing classification scheme under inivestigation [20].

The following work can be divided into two parts. The first is a thorough investigation of the WZW action functional and its application to conformal field theories. The construction of the WZW model is described, followed by a description of the gauging prescription. The parafermionic fields are introduced, and their classical Poisson bracket relations are investigated. A semi-classical treatment of these relations is shown to be consistent with known relations of the coset models. Finally, the recently discovered W-algebra symmetry is shown to arise naturally in this approach.

The second part of this thesis concerns the fascinating connection between the WZW models and the quantization of a three-dimensional "topological" (topological in the sense that the action is independent of the spacetime metric) action functional called the Chern-Simons action. Although much of the information of the conformal model is not explicitly present (eg. descendent field structure, correlation functions, etc.), the Chern-Simons theory gives a simple description of the fusion algebra, leading to a new demonstration of the Verlinde conjecture (that the modular transformation $S: \tau \rightarrow-1 / \tau$ diagonalizes the fusion rules). Finally, the Chern-Simons view is considered in solving some open questions concerning the existence and uniqueness of coset models containing so-called fixed points.

The original work embodied in this thesis has appeared previously in three published articles. The work on the non-abelian gauged WZW model and the 
observance of the W-algebra symmetry was co-authored by my advisor $\mathrm{K}$. Bardakci, my fellow graduate student, Michael Crescimanno, and myself [10, 11]. The work on Chern-Simons theory was co-authored by Michael Crescimanno and myself [23]. I would like to thank them for allowing me to reproduce these results in thesis form. 


\section{Part I}

\section{Parafermions and Conformal Field Theory}

\section{Lagrangian Models in Conformal Field Theories}

The first constructions of conformally invariant field theories were algebraic in nature $[7,14,30,34,49]$. If these models are to be useful in constructing physical models it is desirable that they be given a classical or Lagrangian foundation. One obvious example of this is in the application of conformal field theories to string compactification models. In a seminal paper [58] Witten showed that the quanization of the Wess-Zumino-Witten (WZW) Lagrangian gives rise to a conformally invariant theory, correctly implementing the Sugawara construction and the underlying affine current algebra. The coset constructions, which can be thought of as a generalization of the Sugawara constructions, were then shown to follow from a "gauged" version of the WZW Lagrangian. In fact, the gauged field is not dynamical, but acts as a Lagrange multiplier to project out the currents belonging to a particular subalgebra.

In the non-gauged WZW model it is convenient to formulate the model in terms of conserved currents. As a consequence of conformal invariance in two dimensions there exists two sets of conserved currents. In light-cone coordinates, we have one set of conserved currents independent of the $x_{+}$coordinate and one set independent of the $x_{-}$coordinate. The connection to CFT is made when a careful analysis reveals that these currents form a representation of the affine Lie algebra corresponding to the group in which the classical field takes its values. When constructed in the gauged WZW model, these lncal currents will be gauge dependent, and therefore only covariantly conserved. The fundamental idea of the following work is to construct a new set of gauge invariant currents by attaching Wilson lines to the original gauge dependent currents. These new currents are gauge invariant as well as conserved classically, at the cost of being non-local. We will call these new currents the "classical parafermionic currents" in that they are reminiscent of the parafermionic fields introduced by Zamolod- 
chikov [61].

To construct the quantum theory in terms of the parafermionic currents, the commutation relations between them must be computed. Because the currents are non-local, equal-time commutation relations are ill-defined. Instead we first compute the classical Poisson brackets of the parafermionic currents, which are the classical analogue of the operator product expansion. For abelian coset models (that is, the gauged subalgebra is abelian) the resulting Poisson bracket is quite simple, although the calculation is somewhat laborious. For non-abelian cosets however, a number of difficulties arise. Because of complicater base point contributions, the Possion brackets do not close for non-abelian cosets. These base point contributions are gauge artifacts, and we show that the PBs close among gauge invariant currents (color singlets). Also in the non-ablian coset models, an interesting problem concerning the independence of axial and gauge transformations is addressed.

The fact that the Possion bracket closes for color singlet products of currents, but not for the individual parafermionic currents, gives rise to an interesting interpretation. These color singlet composite fields are the classical analogue of the primary fields. In this sense, the parafermionic currents are analogous to "quark" fields and the primary fields are the composite fields formed from the "quarks". In computing the Poisson bracket of primary fields from that of its constituent "quark" fields, it is observed that the base point dependent terms cancel. It is natural then to consider the Poisson algebra of the "quark" fields without the base point dependent terms. This algebra can be considered to be the generating algebra of the Poisson algebra of the prirnary fields. Because the base point dependent terms have been dropped, this algebra no longer satisfies the Jacobi identity; that is, the algebra is no longer associative. (The loss of associativity is true only for non-abelian cosets). In a natural way then, we have seen how non-associative algebras may play a role in conformal field theory. Compare this to the quasi-Hopf algebra structure of conformal field theory, in which the tensor product operation on algebra representations is non-associative [27].

Certain conformal field theories derived from the coset construction are known to carry an enlarged symmetry algebra called a $W$ algebra $[4,5,15,29,55$, $56,60]$. The $\mathrm{W}$ algebra can be viewed as a natural generalization of the Virasoro 
algebra. It is not surprising then that the classical limit of the $\mathrm{W}$ algebra can be derived from the Poisson algebra discussed above. We demonstrate this connection, and introduce a natural generalization of these classical $\mathrm{W}$ algebras by considering the Poisson algebra of multilocal fields.

After developing these classical Poisson algebras, we present a realization of the parafermion fields which we call the free current construction. For abelian coset models, the realization in free fields has been known for some time $[16,53]$. The free current construction is a generalization of this construction in which the free fields are replaced by currents satisfying the affine Lie algebra. This structure can be inferred by thinking of the Wilson lines attached to the local currents as path ordered exponentials of free fields.

The free current construction suggests a natural method of quantization. We wish to replace the classical free currents satisfying the affine Possion bracket algebra by operators which satisfy the corresponding commutator algebra. This process leads to difficult problems concerning operator renormalization. As a conclusion we discuss some of the many open questions remaining concerning the full quantum theory.

\section{Review of the Wess-Zumino-Witten Model}

We begin with the Wess-Zumino-Wiiten action,

$$
I_{W Z W}=\frac{k}{4 \pi} \int d^{2} x \operatorname{Tr}\left(\partial_{+} g^{-1} \partial_{-} g\right)+\frac{k}{6 \pi} \int d^{3} x \operatorname{Tr}\left(g^{-1} \partial_{+} g g^{-1} \partial_{-} g g^{-1} \partial_{x} g\right)
$$

where $x_{ \pm}=x_{0} \pm x_{1}, \partial_{ \pm}=\frac{1}{2}\left(\partial_{0} \pm \partial_{1}\right), \mathrm{g}(\mathrm{x})$ is a field defined over some twomanifold taking values in a Lie group $\mathrm{G}$, and the trace is taken in some representation of $g$, the Lie algebra of $\mathrm{G}$. We normalize the trace so that $\operatorname{Tr}\left(\tau^{a} \tau^{b}\right)=2 \delta^{a b}$. In computing the second integral, we treat the two dimensional space-time manifold as the boundary of a three-sphere, extending the field $g(x)$ smoothly. The field $\mathrm{g}(\mathrm{x})$ may be extended in topologically distinct ways. The space of topological classes of smooth extensions of the field $g(x)$ is isomorphic to $Z$, the integers, and thus a given extension may be labelled by an integral winding number [25, 26]. The coefficient of the Chern-Simons term has been chosen so that under a change of winding number 1 , the action changes by a factor of $2 \pi k$, and thus the contribution to the path integral is unchanged if and only if $k$ is 
an integer. Therefore, for the path integral to be well-defined, it is necessary that $\mathrm{k}$ satisfy the quantization condition $k \in \mathbf{Z}$. The constraint leading to the coefficient of the first term concerns the anticipated equations of motion and is discussed next.

The variation of $I_{W Z W}$ is a local functional of the two-manifold. In fact, under $g \rightarrow g+\delta g$ we have,

$$
\delta I_{W Z W}=\frac{k}{2 \pi} \int d^{2} x \operatorname{Tr}\left(g^{-1} \delta g \partial_{\mu}\left(g^{-1} \partial_{\mu} g\right)\right)-\frac{k}{2 \pi} \int d^{2} x \operatorname{Tr}\left(g^{-1} \delta g \epsilon^{\mu \nu} \partial_{\mu}\left(g^{-1} \partial_{\nu} g\right)\right) .
$$

Thus the variational equations are given by,

$$
\frac{k}{\pi} \partial_{-}\left(g^{-1} \partial_{+} g\right)=0
$$

Defining the currents,

$$
j_{+}=\frac{i k}{\pi} g^{-1} \partial_{+} g ; j_{-}=-\frac{i k}{\pi}\left(\partial_{-} g\right) g^{-1}
$$

gives the desired result,

$$
\partial_{-} j_{+}=0
$$

Note that the following identity (which holds for arbitrary field $\mathrm{g}(\mathrm{x})$ ),

$$
\partial_{+} j_{-}+\partial_{-} j_{+}=0
$$

along with the above equation of motion gives rise to the partner,

$$
\partial_{+} j_{-}=0 \text {. }
$$

We will see how these equations are modified in the gauged Wess-Zumino-VVitten model.

\section{The Gauged Wess-Zumino-Witten Model}

We would like to add a term to the WZW action that will remove romponents of the currents corresponding to some subgroup $\mathrm{H} \subset \mathrm{G}$. To this end, we will introduce a gauge field $A_{\mu}$, that will act as a non-dynamical Lagrange multiplier. We have,

$$
I=I_{W Z W}+\frac{k}{2 \pi} \int d^{2} x \operatorname{Tr}\left(i A_{+} \partial_{-} g g^{-1}-i A_{-} g^{-1} \partial_{+} g-A_{+} g A_{-} g^{-1}+A_{+} A_{-}\right) .
$$


Note that the last ierm has been added to ensure that the entire action is gauge invariant, that is, that the action is invariant under the transformation:

$$
\begin{aligned}
g(x) & \rightarrow B^{-1}(x) g(x) B(x), \\
A_{\mu}(x) & \rightarrow B^{-1}(x) A_{\mu}(x) B(x)+i B^{-1}(x) \partial_{\mu} B(x),
\end{aligned}
$$

$\mathrm{B}(\mathrm{x}) \in \mathrm{G}$. The currents generating these transformations are the covariant versions of the currents of the WrW model,

$$
J_{+}=\frac{i k}{\pi} g^{-1} D_{+} g ; \quad J_{-}=-\frac{i k}{\pi}\left(D_{-} g\right) g^{-1} .
$$

Where the covariant derivative $D_{\mu}$ is defined by,

$$
D_{\mu} g(x)=\partial_{\mu} g(x)-i\left[A_{\mu}(x), g(x)\right]
$$

Invariance of the action under an arbitrary variation of $A_{+}$and $A_{-}$gives the desired constraint equations:

$$
\operatorname{Tr}\left(h^{a} J_{+}\right)=0=\operatorname{Tr}\left(h^{a} J_{-}\right) \quad \forall h^{a} \in \mathbf{h} .
$$

Where $\mathbf{h}$ is the Lie algebra of $\mathrm{H}$ (a subalgebra of $\mathbf{g}$ ). These equations may be inverted, providing a description of the Lagrange multiplier $A(x)$ in terms of the dynamical field $g(x)$ :

$$
\begin{gathered}
A_{+}^{a}=\frac{i}{2}[1-M]_{a b}^{-1} \operatorname{Tr}\left(g^{-1} \partial_{+} g h^{b}\right), \\
A_{-}^{b}=-\frac{i}{2} \operatorname{Tr}\left(\partial_{-} g g^{-1} h^{a}\right)[1-M]_{a b}^{-1}, \\
M_{a b}=\frac{1}{2} \operatorname{Tr}\left(h^{a} g^{-1} h^{b} g\right) .
\end{gathered}
$$

Where $\mathbf{1}$ is the identity matrix.

The variation of the action under the change $g \rightarrow \delta g$ is given by,

$$
\delta I=\frac{1}{2 i} \int d^{2} x \operatorname{Tr}\left(g^{-1} \delta g\left(D_{-} J_{+}-\frac{k}{\pi} F\right)\right) .
$$

Where the field strength $F$ is defined by,

$$
F=\partial_{+} A_{-}-\partial_{-} A_{+}-i\left[A_{+}, A_{-}\right]
$$

Since the gauge field $A(x)$ takes values only in the subalgebra $\mathrm{h}$, the same must be true for $F(x)$. From eq.(12), we see that the covariant current $J_{+}$takes values in the coset $\mathbf{g}-\mathbf{h}$ and therefore so does the expression $D_{-} J_{+}$. Thus, asking that 
the variation of $I$ vanishes implies the separate cancellation of $\mathrm{F}$ and $D_{-} J_{+}$. We have,

$$
\begin{gathered}
D_{+} J_{-}=0, \\
F=0 .
\end{gathered}
$$

Similar to the ungauged model, we have the following general identity,

$$
D_{+} J_{-}+g D_{-} J_{+} g^{-1}=-\frac{k}{\pi}\left(F-g F g^{-1}\right) \text {. }
$$

Which gives rise to the equation of motion,

$$
D_{+} J_{-}=0
$$

\section{The Parafermionic Currents}

In order to simplify the quantization procedure, we would like to characterize the observable quantities of the theory in terms of chirally conserved currents. We may expect also that these currents take values only in the coset $\mathbf{g}-\mathbf{h}$, and not in the subalgebra $h$. To this end, we introduce the parafermionic currents,

$$
\psi_{\mu}\left(x, x_{0}\right) \equiv-U^{-1}\left(x, x_{0}\right) J_{\mu} U\left(x, x_{0}\right)
$$

with,

$$
U\left(x, x_{0}\right) \equiv P_{C} \exp \left(i \int_{x_{0}}^{x} d x_{\mu}^{\prime} A^{\mu}\left(x^{\prime}\right)\right)
$$

$P_{C}$ represents "path ordering" the exponential along a curve $C$ which connects the base point $x_{0}$ to $x$. In constructing Poisson brackets, we will identify $x_{+}$ with the time coordinate and $x_{-}$with a space coordinate. We will concentrate on the space component of $\psi$, and define,

$$
\psi(x) \equiv \psi_{-}(x)=-U^{-1}\left(x, x_{0}\right) J_{-}(x) U\left(x, x_{0}\right),
$$

where the integration of the path ordered exponentials is carried out at fixed time $\left(x_{+}\right)$. Note that $\mathrm{U}(\mathrm{x}, \mathrm{y})$ satisfies the following important properties,

$$
\begin{array}{ll}
I & U(x, y) U(y, z)=U(x, z), \\
I I & \partial_{x} U(x, y)=i A_{-}(x) U(x, y) ; \partial_{y} U(x, y)=-i U(x, y) A_{-}(y), \\
I I I & \partial_{t} U(x, y)=\int_{y}^{z} d z U(x, z) i \partial_{t} A_{-}(z) U(z, y),
\end{array}
$$


where in the last identily; $t$ refers to the time coordinate, and $x, y$ and $z$ refer to space coordinates.

From the equations of motion (eq.(12)) and the fact that $\psi$ is the conjugation of a coset valued current by an element of the subgroup $H$, we have the desired propertie's,

$$
\begin{gathered}
\operatorname{Tr}\left(h^{a} \psi\right)=0 \quad \forall h^{a} \in \mathrm{h}, \\
\partial_{+} \psi=0 .
\end{gathered}
$$

Physical observables are not dependent on the base point $x_{0}$. A complete set of such variables constructed from the local parafermions is given by the following set of "multilocal" observables,

$$
\begin{aligned}
O\left(x_{1}, x_{2}, \ldots, x_{n}\right) & =\operatorname{Tr}\left(\psi\left(x_{1}\right) \psi\left(x_{2}\right) \cdots \psi\left(x_{n}\right)\right) \\
& =(-1)^{n} \operatorname{Tr}\left(J_{-}\left(x_{1}\right) U\left(x_{1}, x_{2}\right) J_{-}\left(x_{2}\right) \cdots J\left(x_{n}\right) U\left(x_{n}, x_{1}\right)\right) .
\end{aligned}
$$

The term "multilocal" is used in the following sense: the local parafermion has a non-abelian tail, analogous to the Dirac tail of the electromagnetic monopole. When we "tie" these tails together in a gauge invariant manner, as shown above, we remove this non-local dependence. If we were to reinstate the full two-dimensional freedom of these objects, we would find that non-overlapping observables $O_{1}$ and $O_{2}$ would satisfy Bose statistics with respect to one another.

Truly local observables can also be extracted from these observables by letting the space variables approach each other and expanding in the differences $\left(x_{i}-x_{j}\right)$. When we quantize, this procedure will give us the operator product expansion, the coeffiecients of this expansion being identified as the primary fields. In analogy to the quark model, the parafermionic currents are treated as the building blocks of the physical observables.

\section{The Poisson Bracket of the Parafermionic Currents}

In order to quantize the theory, treating the parafermionic currents as local observables, we will need to first compute their classical Poisson bracket. The details of this calculation for the case of abelian cosets is given in ref. [9].

In the case of non-abelian cosets (that is, the subgroup $\mathrm{H}$ is non-abelian), two important complications arise. The first concerns base-point dependent 
terms. These terms result in complicated expressions for the Poisson bracket of the parafermionic currents, which detract from a clear understanding of the algebraic structure. Observable quantities should be independent of the basepoint and, in retrospect, the base-point dependent terms can be dropped in the intermediate calculation. When these terms are dropped, the resulting Poisson algebra for the parafermionic currents is non-associative, associativity being restored only after projecting onto the "multilocal" observables discussed earlier. The second difficulty concerns the linear independence of the components of the field strength tensor on shell. This technical matter can be considered from a pureiy group theoretic viewpoint and is considered below.

We begin by reviewing the computation of Poisson brackets for Lagrangians linear in time derivatives of the field variable. Let,

$$
I=\int d t A_{i}(\phi) \frac{d \phi_{i}}{d t}
$$

The variation of $I$ is given by,

$$
\delta I=\int d t \delta \phi_{i} F_{i j} \frac{d \phi_{j}}{d t}
$$

with,

$$
F_{i j} \equiv \frac{\partial A_{i}}{\partial \phi_{j}}-\frac{\partial A_{j}}{\partial \phi_{i}}
$$

In terms of the variation $\delta \phi_{i}$, the Poisson bracket is given by,

$$
\left\{\delta \phi_{i}, \delta \phi_{j}\right\}=\left(F^{-1}\right)_{i j}
$$

More generally, the following variation in the action,

$$
\delta I=\int d t \delta \phi_{i} V_{j}^{i}(\phi) E_{j k}(\phi) W_{k}^{l}(\phi) \frac{d \phi_{l}}{d t}
$$

gives the Poisson bracket,

$$
\left\{V_{i}, W_{j}\right\}=\left(E^{-1}\right)_{j i}
$$

If the tensor $E$ is not invertible, constraints must be imposed on the dynamical fields. For our action (eq.(8)), invertibility is achieved through gauge fixing. The detailed form of this constraint is not important here since we will be dealing 
only with gauge invariant quantities (up to surface terms at the base point). For example, we will write,

$$
f(x)=U^{-1}\left(x, x_{0}\right) g(x) U\left(x, x_{0}\right),
$$

giving,

$$
\psi=\frac{i k}{\pi}\left(\partial_{-} f\right) f^{-1}
$$

At this point, we need to rewrite the variation of the action in a convenient form and one which will involve only gauge invariant quantities. During the following calculation, the gauge field $A_{\mu}$ and the corresponding field strength terisor $F$, will be treated as functions of $g(x)$ (see eq.(13) and eq.(15).) Define the following variation,

$$
A_{\delta}^{a}=\frac{i}{2}[1-M]_{a b}^{-1} \operatorname{Tr}\left(g^{-1} \delta g h^{b}\right) .
$$

Note that $A_{\delta}$ is simply $A_{+}$with $\partial_{+} g$ replaced by $\delta g$. Recalling our earlier expression for the variation of the action (eq.(14)), we can rewrite the second term in the following way,

$$
\operatorname{Tr}\left(g^{-1} \delta g F\right)=-i \operatorname{Tr}\left(A_{\delta}\left(F-g F g^{-1}\right)\right)
$$

The identity given in eq.(17), along with the fact that the currents $J_{+}$and $J_{-}$ take values only in the coset, gives,

$$
\operatorname{Tr}\left(g^{-1} \delta g F\right)=\frac{i \pi}{k} \operatorname{Tr}\left(g^{-1}\left[A_{\delta}, g\right] D_{-} J_{+}\right)
$$

We have then,

$$
\delta I=\frac{k}{2 \pi} \int d^{2} x \operatorname{Tr}\left(g^{-1}\left(\delta g-i\left[A_{\delta}, g\right]\right)\left(\partial_{-}-i\left[A_{-},\right) g^{-1}\left(\partial_{+} g-i\left[A_{+}, g\right]\right)\right) .\right.
$$

At this point we make the following gauge transformation,

$$
g(x) \rightarrow f(x)=U^{-1}\left(x, x_{0}\right) g(x) U\left(x, x_{0}\right),
$$

with $U\left(x, x_{0}\right)$ defined in eq.(20). Defining,

$$
\begin{aligned}
& H_{+}(x) \equiv-\int^{x} d x^{\prime} U\left(x_{0}, x^{\prime}\right) F\left(x^{\prime}\right) U\left(x^{\prime}, x_{0}\right) \\
& H_{\delta}(x) \equiv-\int^{x} d x^{\prime} U\left(x_{0}, x^{\prime}\right) F_{\delta}\left(x^{\prime}\right) U\left(x^{\prime}, x_{0}\right)
\end{aligned}
$$


Where $F_{\delta}(x)$ is derived from $F(x)$ by replacing $\partial_{+} g(i)$ with $\delta g(x)$. The variation of the action can be rewritten,

$$
\delta I=\frac{k}{2 \pi} \int d^{2} x \operatorname{Tr}\left[f^{-1}\left(\delta f-i\left[H_{\delta}, f\right]\right) \partial_{-}\left(f^{-1}\left(\partial_{+} f-i\left[H_{+}, f\right]\right)\right] .\right.
$$

This variation takes the form of eq.(29). Defining,

$$
R=f^{-1}\left(\partial_{+} f-i\left[H_{\delta}, f\right]\right)=R_{a} \tau^{a} ; \tau^{a} \in \mathbf{g}-\mathbf{h},
$$

we have the Poisson bracket,

$$
\left\{R_{a}(x), R_{b}(y)\right\}=-\frac{\pi}{2 k} \delta_{a b} \epsilon(x-y),
$$

where $\epsilon(x-y)$ is defined by,

$$
\epsilon(x-y) \equiv \begin{cases}+1 & x>y \\ -1 & x<y\end{cases}
$$

Consider the axial transformation,

$$
\delta g=-\frac{i}{2}\left[h^{a}, g\right]_{+} \delta \Theta^{a} ; h^{a} \in \mathbf{h} .
$$

(Here $[,]_{+}$refers to the anti-commutator.) Using eq.(8) and eq.(17), we have,

$$
\delta I=\frac{k}{2 \pi} \int d^{2} x \operatorname{Tr}\left(h^{a} F\right) \delta \Theta^{a},
$$

which gives the Poisson bracket,

$$
\left\{\delta \Theta^{a}(x), F^{b}(y)\right\}=\frac{\pi}{k} \delta_{a b} \delta(x-y) .
$$

These two Poisson brackets (eq.(41) and eq.(45)) will, after some algebraic manipulation, provide us with the Poisson bracket of the parafermionic currents.

Following Witten [58], we work with an index free notation by defining,

$$
\psi_{P} \equiv \int d x \operatorname{Tr}(P(x) \delta \psi(x)), P(x) \in \mathbf{g}-\mathbf{h} .
$$

Using eqs. $(32,40)$, we can rewrite $\psi_{P}$ as,

$$
\begin{aligned}
\psi_{P} & =\frac{i k}{\pi} \int d x \operatorname{Tr}\left(P(x) f \partial_{-}\left(f^{-1} \delta f\right) f^{-1}\right) \\
& =-\frac{i k}{\pi} \int d x \operatorname{Tr}\left(f^{-1} \delta f \partial_{-}\left(f^{-1} P f\right)\right) \\
& =-\frac{i k}{\pi} \int d x \operatorname{Tr}\left(R \partial_{-}\left(f^{-1} P f\right)+i f^{-1}\left[H_{\delta}, f\right] \partial_{-}\left(f^{-1} P f\right)\right)
\end{aligned}
$$


We can break up the Poisson bracket of the parafermionic currents into two parts,

$$
\left\{\psi_{P}(x), \psi_{Q}(y)\right\}=\beta+\gamma
$$

where we have defined,

$$
\begin{gathered}
\beta=-\frac{k^{2}}{\pi^{2}} \int d x d y\left\{\operatorname{Tr}\left(R(x) \partial_{-}\left(f^{-1} P f\right)\right), \operatorname{Tr}\left(R(y) \partial_{-}\left(f^{-1} Q f\right)\right)\right\} \\
\gamma=-\frac{k^{2}}{\pi^{2}} \int d x d y\left\{\operatorname{Tr}\left[\left(R+\frac{i}{2} f^{-1}\left[H_{\delta}, f\right]\right) \partial_{-}\left(f^{-1} P f\right)\right]\right. \\
\left.\operatorname{Tr}\left[i f^{-1}\left[H_{\delta}, f\right] \partial_{-}\left(f^{-1} Q f\right)\right]\right\}
\end{gathered}
$$

Using eq.(41) we can readily compute $\beta$, we have,

$$
\begin{aligned}
\beta= & -\frac{4 k^{2}}{\pi^{2}} \sum_{h^{a}, h^{b} \in \mathbf{g}-\mathbf{h}} \int d x d y\left(\partial_{-}\left(f^{-1} P f\right)\right)_{a}\left(\partial_{-}\left(f^{-1} Q f\right)\right)_{b}\left\{R^{a}(x), R^{b}(y)\right\} \\
= & -\frac{4 k}{\pi} \sum_{h^{a} \in \mathrm{g}-\mathbf{h}} \int d x\left(f^{-1} P f\right)_{a} \partial_{-}\left(f^{-1} Q f\right)_{a} \\
= & \left.\left.\frac{2 k}{\pi} \int d x \operatorname{Tr}\left(f^{-1} Q f\right) \partial_{-} f^{-1} P f\right)\right) \\
& -\frac{k}{\pi} \sum_{h^{a} \in \mathbf{h}} \int d x \operatorname{Tr}\left(h^{a} f^{-1} Q f\right) \operatorname{Tr}\left(h^{a} \partial_{-}\left(f^{-1} P f\right)\right) \\
= & \frac{2 k}{\pi} \int d x \operatorname{Tr}\left(Q \partial_{-} P\right)+2 i \int d x \operatorname{Tr}([P, Q] \psi) \\
& -\frac{k}{\pi} \sum_{h^{a} \in \mathbf{h}} \int d x \operatorname{Tr}\left(h^{a} f^{-1} Q f\right) \operatorname{Tr}\left(h^{a} \partial_{-}\left(f^{-1} P f\right)\right) .
\end{aligned}
$$

To compute $\gamma$, we rewrite the left-hand side of the Poisson bracket as,

$$
\operatorname{Tr}\left[\left(f^{-1} \delta f-\frac{i}{2} f^{-1}\left[H_{\delta}, f\right]\right) \partial_{-}\left(f^{-1} P f\right)\right] .
$$

Employing the equality,

$$
H_{\delta}(x)=U^{-1}\left(x, x_{0}\right) A_{\delta}(x) U\left(x, x_{0}\right)+i U^{-1}\left(x, x_{0}\right) \delta U\left(x, x_{0}\right),
$$

we have the following expression for the left hand side of $\gamma$ under the axial transformation of eq.(43),

$$
\begin{gathered}
\operatorname{Tr}\left[\left(f^{-1} \delta f-\frac{i}{2} f^{-1}\left[H_{\delta}, f\right]\right) \partial_{-}\left(f^{-1} P f\right)\right]=\operatorname{Tr}\left(-\left(\frac{i}{2} f^{-1}\left[f, U^{-1} h^{a} U\right]_{+} d \Theta^{a}\right.\right. \\
\left.-\frac{1}{2} f^{-1}\left[U^{-1} \delta U, f\right]-\frac{i}{2} f^{-1}\left[U^{-1} A_{\delta} U, f\right]\right) .
\end{gathered}
$$

The last two terms of this expression have a relative sign opposite of the two terms of the right hand side of $\gamma$. This relative sign, after antisymmetrizing, results in cancellation. This leaves,

$$
\begin{aligned}
\gamma= & -\frac{k^{2}}{\pi^{2}} \int d x d y\left\{\operatorname{Tr}\left(-\frac{i}{2} f^{-1}\left[f, U^{-1} h^{a} U\right]_{+} d \Theta^{a} \partial_{-}\left(f^{-1} P f\right)\right)\right. \\
& \left.\operatorname{Tr}\left(-i H_{\delta} \partial_{-}\left(f^{-1} Q f\right)-\frac{\pi}{k} H_{\delta}[\psi, Q]\right)\right\}
\end{aligned}
$$


We require the Poisson bracket of $d \Theta^{a}$ with $H_{\delta}^{b}$, which follows from eq.(45), we have,

$$
\left\{d \Theta^{a}(x), H_{\delta}^{b}(y)\right\}=\frac{\pi}{4 k} \operatorname{Tr}\left(U^{-1} h^{a} U h^{b}\right) \epsilon(x-y) .
$$

Combining these gives, after some algebraic manipulation,

$$
\begin{aligned}
\gamma= & \frac{k}{\pi} \sum_{h^{a} \in \mathrm{h}} \int d x \operatorname{Tr}\left(h^{a} f^{-1} Q f\right) \operatorname{Tr}\left(h^{a} \partial_{-}\left(f^{-1} P f\right)\right) \\
& +\frac{\pi}{2 k} \sum_{h^{a} \in \mathbf{h}} \int d x d y \operatorname{Tr}\left(\left[h^{a}, P\right] \psi\right) \epsilon(x-y) \operatorname{Tr}\left(\left[h^{a}, Q\right] \psi\right) .
\end{aligned}
$$

The first term cancels with the third term of $\beta$, and finally,

$$
\begin{aligned}
\left\{\psi_{P}, \psi_{Q}\right\}= & \frac{2 k}{\pi} \int \operatorname{Tr}\left(Q \partial_{-} P\right)+2 i \int \operatorname{Tr}([P, Q] \psi) \\
& +\frac{\pi}{2 k} \sum_{h^{a} \in \mathbf{h}} \int d x d y \operatorname{Tr}\left(\left[h^{a}, P\right] \psi\right) \epsilon(x-y) \operatorname{Tr}\left(\left[h^{a}, Q\right] \psi\right) .
\end{aligned}
$$

\section{The Poisson Algebra and Multilocal Observables}

The Poisson algebra of the Parafermionic currents derived in the previous section is remarkably simple, considering the lengthy derivation. In this section, we will consider the structure of this algebra and its extension to multilocal observables.

The Poisson algebra presented in eq.(57) should satisfy the Jacobi identity,

$$
\sum_{\text {cyclic }}\left\{\left\{\psi_{P_{1}}\left(x_{1}\right), \psi_{P_{2}}\left(x_{2}\right)\right\}, \psi_{P_{3}}\left(x_{3}\right)\right\}=0
$$

the sum being taken over cyclic permutations of the three currents. This follows from the fact that the Jacobi identity is preserved under canonical transformations. Under direct calculation, however, we find that the identity is not satisfied in general for the case of non-abelian subgroup $\mathrm{H}$. The error in our calculation can be traced back to the derivation of eq.(45) from eq.(44). This step is valid if and only if the components of $F$ are linearly independent, that is, only if there is no linear constraint of the form,

$$
\sum_{a} F^{a}(x) C_{a}(g(x))=0
$$

Note that $C_{a}$ may depend on $g(x)$ but not $\partial_{t} g(x)$. If such relations exist, we have a constrained system, and the right hand side of eq. (45) must be modified to,

$$
\left\{d \Theta^{a}(x), F^{b}(y)\right\}=\frac{\pi}{k} \delta(x-y) P^{a b}
$$


where $P^{a b}$ is the projection of the unit operator $\delta^{a b}$ in the subspace orthogonal to the constraints. The existence of such a constraint can be seen from a purely group theoretical point of view. In addition to the axial transformations of eq.(43), consider the infinitesimal vector (gauge) transformations,

$$
\delta_{V} g=-\frac{i}{2}\left[h^{a}, g\right] d \chi^{a} .
$$

The existence of a linear relation between $\delta_{V} g$ and $\delta_{A} g$ would imply that a certain linear combination of axial transformations is equivalent to a gauge transformation, which leaves the action unchanged. Since $d \Theta$ is arbitrary, this implies a linear relation on $\mathrm{F}$ of the form eq.(59). If $\mathbf{h}$ is the Cartan subalgebra of $g$ then no such relations exist, and eq.(45) is unchanged. This can be proved simply by diagonalizing $\mathrm{h}$ and writing out such a linear relation on components. However, if $\mathbf{h}$ is non-abelian the situation is quite different. We consider the case: $\mathrm{G}=$ $\mathrm{SU}(2) \otimes \mathrm{SU}(2)$, and $\mathrm{H}=\mathrm{SU}(2)$ (diagonal subgroup). If we take $\mathrm{SU}(2)$ in the fundamental representation, we can parameterize $\left(g_{1}, g_{2}\right)$ in the following way,

$$
\begin{gathered}
g_{1}=a_{0} I_{1}+i \vec{a} \cdot \vec{\sigma}_{1} ; g_{2}=b_{0} I_{2}+i \vec{b} \cdot \vec{\sigma}_{2} \\
\text { with }\left(a_{0}\right)^{2}+(\vec{a})^{2}=1 ;\left(b_{0}\right)^{2}+(\vec{b})^{2}=1
\end{gathered}
$$

Here $\sigma$ represents the Pauli matrices and $I$ the unit matrix. In this notation, we can parameterize the subalgebra $\mathbf{h}$ by,

$$
h=\vec{h} \cdot\left(\vec{\sigma}_{1}+\vec{\sigma}_{2}\right) ; h \in \mathbf{h} .
$$

The desired linear relation is given by,

$$
\begin{gathered}
{\left[g_{1} \otimes g_{2}, \vec{n} \cdot\left(\vec{\sigma}_{1}+\vec{\sigma}_{2}\right)\right]_{+}=\left[g_{1} \otimes g_{2}, \vec{m} \cdot\left(\vec{\sigma}_{1}+\vec{\sigma}_{2}\right)\right]} \\
\vec{n}=\frac{\vec{a} \times \vec{b}}{|\vec{a} \times \vec{b}|} ; \vec{m}=\frac{b_{0} \vec{a}-a_{0} \vec{b}}{|\vec{a} \times \vec{b}|} .
\end{gathered}
$$

It follows that,

$$
\begin{gathered}
\sum_{a=1}^{3} n^{a} F^{a}=0 \\
\left\{d \Theta^{a}(x), F^{b}(y)\right\}=\frac{\pi}{k} \delta(x-y)\left(\delta^{a b}-n^{a} n^{b}\right) .
\end{gathered}
$$

The existence of linear relations of this type, in many cases, follow from simple counting arguments. For example, consider the coset SU(5)/SU(4). The dimension of $\mathrm{SU}(5)$ is 24 , while for generic $\mathrm{g}$ the rank of $\delta_{A}$ and $\delta_{V}$ are 15 and 12 
respectively. Since $15+12>24$, linear relations must exist. It is not true that such degeneracies exist for all non-abelian cosets; however, they were found in most of the cases we studied.

The modification of the Poisson bracket of $d \Theta$ and $F$ changes the computation of the Poisson bracket of the parafermions in a subtle way. This modification tells us that a subset of chiral transformations is made up of gauge transformations, and we are instructed to project out these variations in the Poisson bracket relations. Such a projection is unnecessary when computing Poisson brackets between gauge invariant quantities. For example, both $R$ and $H_{\delta}$ are formally gauge-invariant. When we coinpute the Poisson bracket of $R$ and $H_{\delta}$ with $F$, we isolate the part of $R$ and $H_{\delta}$ corresponding to chiral variations $d \Theta$. However, if those chiral variations are along a gauge direction, then the corresponding variation of $R$ and $H_{\delta}$ is zero by gauge invariance. This would seem to indicate that the naive calculation based on eq.(45) was correct. However $R, H_{\delta}$ and $\psi$ are not completely gauge invariant; rather, there is gauge dependence at the base point $x_{0}$. When computing the Poisson bracket, base point contributions arise that depend on the projection operator $\mathrm{P}$ of eq.(60). We do not compute these extra terms explicitly, however, since the advantage of working solely with the one-body $\psi s$ is lost.

We can avoid these complications by recalling that ultimately we are interested only in the truly gauge invariant observables of eq.(24). In computing the Poisson bracket of two such observables, the base point contributions cancel. It follows that although eq.(57) is not correct as it stands for non-abelian cosets, it is still perfectly all right to use it as an intermediate step in computing the Poisson bracket of gauge invariant quantities, such as those given in eq.(24).

To conclude this section, we verify that the Jacobi identity is satisfied for the multilocal observables defined in eq.(24). When we substitute the Poisson bracket of eq.(57) into eq.(58), we get an expansion in $k^{i}$, where $i$ takes values in the set, $i \in\{-2,-1,0,1,2\}$. It is not difficult to show that all these terms cancel, with the exception of $i=0$ and $i=-2$. The term independent of $\mathrm{k}$ $(i=0)$ has two contributions: one term where the $\mathrm{k}$-independent term of the Poisson bracket is used twice and one where the non-local term proportional to $\epsilon(x-y)$ is in the inner Poison bracket and the "central charge" term is in the outer Poisson bracket. These terms combine to give a restatement of the Jacobi 
identity for the underlying semi-simple Lie algebra, and in this manner give a non-trivial restriction on the coefficients of the Poisson bracket of eq.(57). If we label the coefficients of the three terms of eq.(57) as $a, b$ and $c$ respectively, this restriction takes the form,

$$
4 a c=-b^{2}
$$

which is indeed satisfied. Letting this expression fix $c$ in terms of $a$ and $b$, we can arbitrarily scale the parafermions $\psi$ to fix $b$. This leaves an overall normalization constant for the right hand side of the Poisson algebra. We now concentrate on the leading term $(i=-2)$ and work with the truncated algebra,

$$
\left\{\psi_{P}(x), \psi_{Q}(y)\right\}=\int d x d y \epsilon(x-y) \sum_{a} \operatorname{Tr}\left(P(x)\left[h^{a}, \psi(x)\right]\right) \operatorname{Tr}\left(Q(y)\left[h^{a}, \psi(y)\right]\right),
$$

the sum being carried out over the subalgebra h. With $O(x)=O\left(x_{1}, x_{2}, \ldots, x_{n}\right)$ defined in eq.(24), we wish to show that,

$$
\sum_{\text {cyclic }}\left\{\left\{O_{1}(x), O_{2}(y)\right\}, O_{3}(z)\right\}=0
$$

Define,

$$
\begin{gathered}
X_{i}^{a} \equiv \operatorname{Tr}\left(\psi\left(x_{1}\right) \cdots\left[h^{a}, \psi\left(x_{i}\right)\right] \cdots \psi\left(x_{n}\right)\right), \\
X_{i j}^{a b} \equiv\left\{\begin{array}{l}
\operatorname{Tr}\left(\psi\left(x_{1} \cdots\left[h^{a}, \psi\left(x_{i}\right)\right] \cdots\left[h^{b}, \psi\left(x_{j}\right)\right] \cdots \psi\left(x_{n}\right)\right) i \neq j\right. \\
\operatorname{Tr}\left(\psi\left(x_{1}\right) \cdots\left[h^{a},\left[h^{b}, \psi\left(x_{i}\right)\right]\right] \cdots \psi\left(x_{n}\right)\right) i=j,
\end{array}\right.
\end{gathered}
$$

and similasly for $\mathrm{Y}$ and Z. Note that $X_{i j}^{a b}$ satisfies the identity,

$$
X_{i j}^{a b}-X_{j i}^{b a}=\delta_{i j} f_{a b c} X_{i}^{c} ;\left(\left[h^{a}, h^{b}\right]=f_{a b c} h^{c}\right) .
$$

We have,

$$
\left\{O_{1}(x), O_{2}(y)\right\}=\epsilon(x-y) X_{i}^{a} Y_{j}^{a},
$$

where the index " $a$ " is summed over the subalgebra $h$, and the indices " $i$ " and " $j$ " are summed over the multivariable index range of " $x$ " and " $y$ " respectively. Taking the bracket with $\mathrm{O}_{3}(z)$ gives,

$$
\left.\left\{O_{1}(x), O_{2}(y)\right\}, O_{3}(z)\right\}=\epsilon\left(x_{i}-y_{j}\right)\left(X_{i l}^{a b} Y_{j}^{a} Z_{k}^{b} \epsilon\left(x_{l}-x_{k}\right)+X_{i}^{a} Y_{j l}^{a b} Z_{k}^{b} \epsilon\left(y_{l}-z_{k}\right)\right) .
$$


Since we are summing over permutations, we are free to permute the second term of eq.(73) twice in $(x, y, z)$. After rearranging dummy indices and employing eq. (71), this expression reduces to,

$$
f_{a b c} X_{i}^{2} Y_{j}^{b} Z_{k}^{c} \epsilon\left(x_{i}-y_{j}\right) \epsilon\left(x_{i}-z_{i:}\right)
$$

We sum permutations and rewrite dummy indices to arrive at,

$$
\begin{gathered}
\sum_{\text {cydic }}\left\{\left\{O_{1}(x), O_{2}(y)\right\}, O_{3}^{\prime}(z)\right\}=f_{a b c} X_{i}^{a} Y_{j}^{b} Z_{k}^{c}\left(\epsilon\left(x_{i}-y_{j}\right) \epsilon\left(x_{i}-z_{k}\right)\right. \\
\left.+\epsilon\left(y_{j}-z_{k}\right) \epsilon\left(y_{j}-x_{i}\right)+\epsilon\left(z_{k}-x_{i}\right) \epsilon\left(z_{k}-y_{j}\right)\right)
\end{gathered}
$$

Employing the :dentity,

$$
\epsilon(x-y) \epsilon(x-z)+\epsilon(y-z) \epsilon(y-x)+\epsilon(z-x) \epsilon(z-y)=1 \forall x, y, z \in \mathbf{R},
$$

gives,

$$
\sum_{\text {cyclic }}\left\{\left\{O_{1}(x), O_{2}(y)\right\}, O_{3}(z)\right\}=\sum_{i, j, k} f_{a b c} X_{i}^{a} Y_{j}^{b} Z_{k}^{c}=\sum_{j, k} f_{a b c} Y_{j}^{b} Z_{k}^{c} \sum_{i} X_{i}^{a}
$$

By color neutrality, the sum over "i" on the right hand side of eq.(77) vanishes, giving the desired result. Notice that for the Jacobi identity to be satisfied, it is only necessary that at least one of the observables be a color singlet.

\section{The Classical W Algebras}

We wish to generalize this procedure to include coset $\mathrm{g} / \mathrm{h}$ models of a very particular type: we take $g$ to be the direct sum of two copies of an affine Lie algebra with arbitrary central charges $k_{1}$ and $k_{2}$ and take $\mathrm{h}$ to be the diagonal subalgebra,

$$
\mathbf{g}=\mathbf{g}\left(k_{1}\right) \oplus \mathbf{g}\left(k_{2}\right), \mathbf{h}=\mathbf{g}\left(k_{1}+k_{2}\right)_{\text {diagonal }} .
$$

This choice is dictated solely by the fact that these cosets have been discussed at length in many previous works $[4,5,6,17]$. Much of this section can be easily generalized to other types of coset models. The gauged WZW action 
corresponding to the coset of eq.(78) is,

$$
\begin{gathered}
I=I_{1}+I_{2}+I_{1,2} \\
I_{1}=\frac{k_{1}}{4 \pi} \int d^{2} x \operatorname{Tr}\left(\partial_{+} g_{1}^{-1} \partial_{-} g_{1}\right)+\frac{k_{1}}{24 \pi} \int \operatorname{Tr}\left(d g_{1} g_{1}^{-1}\right)^{3}, \\
I_{2}=\frac{k_{2}}{4 \pi} \int d^{2} x \operatorname{Tr}\left(\partial_{+} g_{2}^{-1} \partial_{-} g_{2}\right)+\frac{k_{2}}{24 \pi} \int \operatorname{Tr}\left(d g_{2} g_{2}^{-1}\right)^{3}, \\
I_{1,2}=\frac{1}{8 \pi} \int d^{2} x \operatorname{Tr}\left(i A_{+}\left(k_{1}\left(\partial_{-} g_{1}\right) g_{1}^{-1}+k_{2}\left(\partial_{-} g_{2}\right) g_{2}^{-1}\right)\right. \\
-i A_{-}\left(k_{1} g_{1}^{-1}\left(\partial_{+} g_{1}\right)+k_{2} g_{2}^{-1}\left(\partial_{+} g_{2}\right)\right)-k_{1} A_{+} g_{1} A_{-} g_{1}^{-1} \\
\left.-k_{2} A_{+} g_{2} A_{-} g_{2}^{-1}-\left(k_{1}+k_{2}\right) A_{+} A_{-}\right) .
\end{gathered}
$$

Note that the gauge field $A_{\mu}$ takes values in the diagonal subalgebra $\mathbf{h}$. The currents of the model are given by,

$$
\begin{aligned}
& J_{+}=\frac{i k_{1}}{\pi} g_{1}^{-1} D_{+} g_{1}+\frac{i k_{2}}{\pi} g_{2}^{-1} D_{+} g_{2}, \\
& J_{-}=-\frac{i k_{1}}{\pi}\left(D_{-} g_{1}\right) g_{1}^{-1}-\frac{i k_{2}}{\pi}\left(D_{-} g_{2}\right) g_{2}^{-1},
\end{aligned}
$$

with $D_{\mu}$, the covariant derivative, as defined in eq.(11). As before, these currents are not chirally conserved. We define,

$$
\psi \equiv \psi_{-}=-U^{-1}\left(x, x_{0}\right) J_{-}(x) U\left(x, x_{0}\right),
$$

with $U\left(x, x_{0}\right)$, the Wilson line, defined as in eq.(20). The parafermionic current $\psi(x)$ is chirally conserved $\left(\partial_{+} \psi=0\right)$ and gauge invariant up to base point dependent terms. The computation of the Poisson bracket of the parafermionic currents is similar to the derivation given in Section 6, and we will simply present the result,

$$
\begin{aligned}
\left\{\psi^{a}(x), \psi^{b}(y)\right\}= & -\frac{2 k_{1} k_{2}}{\pi\left(k_{1}+k_{2}\right)} \delta^{\prime}(x-y)+\frac{4\left(k_{1}-k_{2}\right)}{\left(k_{1}+k_{2}\right)} \delta(x-y) f_{a b c} \psi^{c}(x) \\
& -\frac{4 \pi}{\left(k_{1}+k_{2}\right)} \epsilon(x-y) f_{a d_{c}} f_{b e c} \psi^{d}(x) \psi^{e}(y) .
\end{aligned}
$$

Where we have defined,

$$
\psi(x)=\psi^{a}(x) \tau^{a}=\psi^{a}(x)\left(\tau_{1}^{a}-\tau_{2}^{a}\right) ;\left[\tau_{1}^{a}, \tau_{1}^{b}\right]=f_{a b c} \tau_{1}^{c} .
$$

As in our earlier calculation, dropping base point dependent terms has rendered this algebra non-associative (that is, the Jacobi identity is not satisfied), a property which is restored only after projecting onto color-singlet observables.

The simplest neutral combination one can form is obtained by taking the trace of an arbitrary power of $\psi$. Compactifying to the circle and scaling appropriately to conform to the literature we define,

$$
W_{m}^{(s)} \equiv \frac{1}{i a s} \int_{0}^{2 \pi} d x \exp (i m x) \operatorname{Tr}\left(\psi^{s}(x)\right)
$$


Where we have defined the constant,

$$
a \equiv \frac{4 k_{1} k_{2}}{\pi\left(k_{1}+k_{2}\right)}
$$

For concreteness we take $g=S U(N)$. We compute the Poisson brackets of the operators in eq.(84) using eq.(82),

$$
\begin{gathered}
\left\{W_{m}^{(s)}, W_{n}^{(t)}\right\}=(m(t-1)-n(s-1)) W_{m+n}^{(s+t-2)} \\
-\frac{i a(s-1)(t-1)}{2 \pi N} \sum_{j} j W_{m-j}^{(s-1)} W_{n+j}^{(t-1)}
\end{gathered}
$$

In the limit $N \rightarrow \infty$ only the first term survives and we have the $\mathrm{SU}(\infty)$ classical W algebra,

$$
\left\{W_{m}^{(s)}, W_{n}^{(t)}\right\}=(m(t-1)-n(s-1)) W_{m+n}^{(s+t-2)} .
$$

For finite values of $\mathrm{N}$ both terms are present and furthermore all $W^{(s)}, \mathrm{s}>\mathrm{N}$ can be expressed in terms of $W^{(s)}, 2 \leq \mathrm{s} \leq \mathrm{n}$. For example in the case of SU(3), we have that,

$$
W_{m+n}^{(4)}=\frac{i a}{4 \pi} \sum_{j} W_{m-j}^{(2)} W_{n+j}^{(2)},
$$

which follows from the trace identity,

$$
\operatorname{Tr}\left(\psi^{4}\right)=\frac{1}{2}\left(\operatorname{Tr}\left(\psi^{2}\right)\right)^{2}
$$

valid for traceless $3 \times 3$ matrices. We therefore find the closed algebra,

$$
\begin{aligned}
& \left\{W_{m}^{(2)}, W_{n}^{(2)}\right\}=(m-n) W_{m+n}^{(2)}, \\
& \left\{W_{m}^{(2)}, W_{n}^{(3)}\right\}=(2 m-n) W_{m+n}^{(3)} \\
& \left\{W_{m}^{(3)}, W_{n}^{(3)}\right\}=\frac{i a}{6 \pi}(m-n) \sum_{l} W_{m-l}^{(2)} W_{n+l}^{(2)} .
\end{aligned}
$$

This agrees with the classical limit of the quantum $W_{3}$ algebra in which one retains only the term with the least singular short-distance behavior. Similarly, closed algebras can be deduced for $W_{N}, N>3$. We note that these algebras have the simple universal form given in ref. [15]. For finite $N$, we see that the $W_{N}$ algebra is nonlinear. This is simply due to structure constant identities used to reduce algebra elements $W^{(s)}$, for $s>N$. It is interesting to note that these algebras are insensitive to the second two terms of the algebra given in eq.(82). We now turn to a generalized algebra without this property. 
We discuss a generalization of $\mathrm{W}$ algebras that is suggested by analyzing WZW models in terms of parafermionic currents. As before, the Poisson algebra of eq.(82) may be used to compute the Poisson bracket of gauge invariant, multilocal observables. In an effort to simplify the resulting algebra, we define a new basis for the multilocal observables (compare eq.(24)),

$$
W\left(x_{1}, x_{2}, \ldots x_{n}\right) \equiv \operatorname{Tr}\left(\psi\left(x_{1}\right)\left[\psi\left(x_{2}\right),\left[\psi\left(x_{3}\right), \ldots \psi\left(x_{n}\right)\right] \ldots\right]\right)
$$

This is a natural gereralization of eq.(84).

We now illustrate this non-local algebra with a simple example. Consider the coset $\mathrm{g}=\left(S U(2)_{k_{1}} X S U(2)_{i_{2}}\right) / S U(2)_{k_{1}+k_{2}}$. In this case, the algebra closes on the first two observables,

$$
\begin{aligned}
& W\left(x_{1}, x_{2}\right)=\operatorname{Tr}\left(\psi\left(x_{1} \psi\left(x_{2}\right)\right),\right. \\
& W\left(x_{1}, x_{2}, x_{3}\right)=\operatorname{Tr}\left(\psi\left(x_{1}\left[\psi\left(x_{2}\right) \psi\left(x_{3}\right)\right]\right) .\right.
\end{aligned}
$$

We have the following algebra,

$$
\begin{aligned}
\left\{W\left(x_{1}, x_{2}\right), W\left(y_{1}, y_{2}\right)\right\}= & -a \delta^{\prime}\left(x_{1}-y_{1}\right) W\left(x_{2}, y_{2}\right)+\text { perm. } \\
& -i \delta \delta\left(x_{1}-y_{1}\right) W\left(x_{1}, x_{2}, y_{2}\right)+\text { perm. } \\
& +2 c \partial\left(x_{1}, x_{2} ; y_{1}, y_{2}\right) W\left(x_{1}, x_{2}, y_{1}, y_{2}\right) \\
\left\{W\left(x_{1}, x_{2}\right), W\left(y_{1}, y_{2}, y_{3}\right)\right\}= & -a \delta^{\prime}\left(x_{1}-y_{1}\right) W\left(x_{2}, y_{2}, y_{3}\right)+\text { perm. } \\
& -i b \delta\left(x_{1}-y_{1}\right) W\left(x_{1}, x_{2}, y_{2}, y_{3}\right)+\text { perm. } \\
& +2 c \theta\left(x_{1}, x_{2} ; y_{1}, y_{3}\right) W\left(x_{1}, x_{2}, y_{1}, y_{2}, y_{3}\right) \\
+ & 2 c \theta\left(x_{1}, x_{2} ; y_{2}, y_{3}\right) W\left(x_{1}, x_{2}, y_{2}, y_{3}, y_{1}\right) \\
\left\{W\left(x_{1}, \dot{w}_{2}, x_{3}, W\left(y_{1}, y_{2}, y_{3}\right)\right\}=\right. & -a \delta^{\prime}\left(x_{1}-y_{1}\right) W\left(x_{2}, x_{3}, y_{2}, y_{3}\right)+\text { perm. } \\
& \left.-i b \delta^{\prime} x_{1}-y_{1}\right) W\left(x_{2}, x_{3}, y_{1}, y_{2}, y_{3}\right)+\text { perm } \\
& +2 c \theta\left(x_{1}, x_{3} ; y_{1}, y_{3}\right) W\left(x_{3}, x_{2}, x_{1}, y_{1}, y_{2}, y_{3}\right) \\
& +2 c \theta\left(x_{2}, x_{3} ; y_{1}, y_{3}\right) W\left(x_{2}, x_{3}, x_{1}, y_{1}, y_{2}, y_{3}\right) \\
& +2 c \theta\left(x_{1}, x_{3} ; y_{2}, y_{3}\right) W\left(x_{3}, x_{2}, x_{1}, y_{2}, y_{3}, y_{1}\right) \\
& +2 c \theta\left(x_{2}, x_{3} ; y_{2}, y_{3}\right) W\left(x_{2}, x_{3}, x_{1}, y_{2}, y_{3}, y_{1}\right)
\end{aligned}
$$

We have defined for convenience the constants,

$$
b \equiv \frac{4\left(k_{1}-k_{2}\right)}{\left(k_{1}+k_{2}\right)} ; c \equiv \frac{2 \pi}{\left(k_{1}+k_{2}\right)},
$$


and have indicated by "perm." the terms obtained by symmetrizing or antisymmetrizing with respect to the arguments in the appropriate way. Note that $W\left(x_{1}, x_{2}\right)$ is symmetric in $x_{1}$ and $x_{2}$ and that $W\left(x_{1}, x_{2}, x_{3}\right)$ is antisymmetric in $x_{1}, x_{2}$, and $x_{3}$. The $\theta$ function is defined by,

$$
\begin{gathered}
2 \theta\left(x_{1}, x_{2} ; y_{1}, y_{2}\right)=\epsilon\left(x_{1}-y_{1}\right)+\epsilon\left(x_{2}-y_{2}\right) \\
-\epsilon\left(x_{1}-y_{2}\right)-\epsilon\left(x_{2}-y_{1}\right)
\end{gathered}
$$

and it measures the overlap between the intervals $\left(x_{1}, x_{2}\right)$ and $\left(y_{1}, y_{2}\right)$. If one interval contains the other or they are disjoint, it vanishes. If they overlap, it is \pm 1 depending on the sense of the overlap. It is simple to extract a truly local algebra from the multilocal observables by expanding in power series in the differences of arguments, as in the operator product expansion.

Finally, we need to show that the functions appearing on the right hand side of eqs.(93-95) can be written in terms of the functions given in eq.(92). This follows from elementary identities between $\mathrm{SU}(2)$ structure constants. We need,

$$
\begin{aligned}
& W\left(x_{1}, x_{2}, x_{3}, x_{4}\right)=\frac{1}{2} W\left(x_{2}, x_{3}\right) W\left(x_{1}, x_{4}\right) \\
& -\frac{1}{2} W\left(x_{1}, x_{3}\right) W\left(x_{2}, x_{4}\right) \text {, } \\
& W\left(x_{1}, x_{2}, x_{3}, x_{4}, x_{5}\right)=\frac{1}{2} W\left(x_{3}, x_{4}\right) W\left(x_{2}, x_{5}, x_{1}\right) \\
& -\frac{1}{2} W\left(x_{3}, x_{5}\right) W\left(x_{2}, x_{4}, x_{1}\right) \text {, } \\
& W\left(x_{1}, x_{2}, x_{3}, x_{4}, x_{5}, x_{6}\right)=\frac{1}{4} W\left(x_{1}, x_{3}\right) W\left(x_{4}, x_{6}\right) W\left(x_{2}, x_{5}\right) \\
& +\frac{1}{4} W\left(x_{2}, x_{3}\right) W\left(x_{4}, x_{5}\right) W\left(x_{1}, x_{6}\right) \\
& -\frac{1}{4} W\left(x_{2}, x_{6}\right) W\left(x_{4}, x_{5}\right) W\left(x_{1}, x_{3}\right) \\
& -\frac{1}{4} W\left(x_{1}, x_{5}\right) W\left(x_{4}, x_{6}\right) W\left(x_{2}, x_{3}\right) \text {. }
\end{aligned}
$$

Using these equalities it is possible to express eqs.(93-95) as a closed algebra. It should not be difficult to write down similar algebras for coset models based on other groups. These algebras (like their local counterparts) take on their complicated, nonlinear structure due to these types of reduction identities.

\section{9 "Free Current" Realization of Parafermion Algebra}

We would like to realize the classical algebras given in eq.(57) in terms of free fields. We will do this in a two step process. First, we will realize these algebras 
in terms of a free current algebra, or affine algebra. The second step is the well-known realization of affine Lie algebras by free fields $[16,53]$ : that is, we give an explicit construction of the currents of the coset models in terms of the currents of the ungauged WZW model. This construction is very simple, and it opens the way for the construction of the full quantum theory $[12,13]$.

We want to find an explicit construction for $\psi$ in terms of fields that satisfy simple, local commutation relations (or Poisson brackets). We mimic eq.(19) by writing,

$$
\psi(x)=V^{-1}\left(x, x_{0}\right) E(x) V\left(x, x_{0}\right),
$$

where $V$ replaces $U$ and $E$ replaces $J . V$ is a bilocal field valued in the subgroup $\mathrm{H}$, defined as a path ordered product, just as in eq.(20),

$$
V\left(x, x_{0}\right)=P_{C} \exp \left(i \int_{x_{0}}^{x} d x^{\prime} B\left(x^{\prime}\right)\right) .
$$

We will require $B$ and $E$ to have local commutation relations. The non-locality of eq.(57) will be entirely due to the non-locality of the Wilson line $V$.

It is possible to avoid path-dependent terms in the commutation relations of the parafermions expressed in eq.(101) if we require $B$ and $E$ to satisfy an affine algebra with correctly chosen central charge. We introduce the free current,

$$
T(x)=\tau^{a} T^{a}(x) ; \tau^{a} \in \mathbf{g}
$$

with the following commutation relations,

$$
\left[T^{a}(x), T^{b}(y)\right]=-\frac{\alpha}{2} \delta^{\prime}(x-y) \delta_{a b}-\frac{i}{4} \delta(x-y) \operatorname{Tr}\left(\left[\tau^{a}, \tau^{b}\right] T(x)\right) .
$$

Define,

$$
T_{(h)} \equiv T^{a} h^{a}, h^{a} \in \mathbf{h} ; T_{(\mathrm{c})} \equiv T^{l} \tau^{l}, \tau^{l} \in \mathbf{g}-\mathbf{h} .
$$

With these currents defined, we identify,

$$
B=\beta T_{(h)} ; E=\gamma T_{(c)},
$$

with the constants $\alpha, \beta$ and $\gamma$ to be determined. Upon computing the commutation relations of the parafermions defined in eq.(101), many base point dependent terms will arise. As before, these terms vanish when projected onto 
color singlet observables, and we ignore them. After some calculation we have the result,

$$
\begin{gathered}
{\left[\psi_{P}(x), \psi_{Q}(y)\right]=\gamma^{2} \alpha \int d x \operatorname{Tr}\left(Q(x) \partial_{x} P(x)-i \gamma \int d x \operatorname{Tr}([P(x), Q(x)] \psi(x))\right.} \\
+\frac{1}{4 \alpha} \int d x d y \epsilon(x-y) \operatorname{Tr}\left(\left[h^{a}, P(x)\right] \psi(x)\right) \operatorname{Tr}\left(\left[h^{a}, Q(y)\right] \psi(y)\right),
\end{gathered}
$$

with the important constraint $\alpha \beta=1$. Taking $\alpha=k / 2 \pi$ and $\gamma=-2$, we have complete agreement with eq.(57). Note that the central charge of the affine Lie algebra eq.(104) is the usual quantized value $k / 2 \pi, k \in \mathrm{Z}$, as required by unitarity. Also note that this construction can easily be extended to the model defined in eq.(79) by employing the tensor sum of two commuting affine Lie algebras [10].

In closing this section, we check two simple consequences of our construction. Consider the gauge invariant quantity,

$$
O(x, y)=\operatorname{Tr}(\psi(x) \psi(y))=4 \operatorname{Tr}\left(T_{(c)}(x) V(x, y) T_{(c)}(y) V(y, x)\right) .
$$

In an earlier work [9], it was shown that the classical stress tensor should be recovered from $O(x, y)$ in the limit $x \rightarrow y$. This is the classical analogue of the leading term in the operator product expansion. From eq.(101), we have,

$$
O(x, x) \approx 8 \sum_{l \in \text { coset }} T^{l}(x) T^{l}(x) .
$$

Up to an overall normalization constant, this is the classical analogue of the Sugawara construction for the coset model.

One other check is to verify that all gauge invariant observables lie in the coset. In an operator language, they should commute with currents that belong to the subgroup $H$. The classical analogue is the following relation,

$$
\left\{O(x, y), T_{(h)}(z)\right\}=0 .
$$

This can easily be shown to hold and in a straightforward way can be generalized to all observables $O\left(x_{1}, \ldots x_{n}\right)$ defined in eq.(24).

\section{Quantization of the Parafermionic Current Algebra}

To make a direct connection with previous work in the theory of two-dimensional coset models, we would like to find a consistent quantum generalization of the 
classical Poisson bracket algebra derived in Section 6. Furthermore, we would like to outline a scheme in which the known spectrum of primary fields may be reproduced, as well as their conformal dimensions and fusion algebra.

Work in this direction has led to many interesting questions concerning braid statistics and quasi-Hopf algebras; see ref.[12, 13] for recent progress in this direction. The full primary field spectrum remains illusory, however. A completion of this scheme holds many rewards, one of which I will now discuss.

In the second part of this thesis, a scheme is outlined whereby one may compute the fusion algebra for the WZW model by employing symmetry properties of the Chern-Simons Hilbert space. Another unsolved problem is the complete extension of these methods to coset models. There exists a class of coset models, those with "fixed points," which have resisted this calculation scheme. In fact, it appears that from this point of view these models allow some freedom in computing the primary field spectrum. If completed, the quantization of the parafermionic current algebra could solve this important mystery. 


\section{Part II}

\section{The Chern-Simons Functional and Conformal Field Theory}

\section{2D Conformal Field Theory and Topological Field Theory}

The axiomatic approach to 2D Conformal Field Theory, pioneered by Belavin, et. al. [14], has led to a rich system of constraints through which we define a rational conformal field theory (RCFT). One interesting aspect of this structure concerns the monodromy, or braiding, constraints of the n-point functions. A connection between these constraints and the defining relations of knot invariants was noticed early on. Knot invariants are purely topological quantities ascribed to objects living in three dimensions. A natural question then, posed and finally solved by Witten [59]; what is the connection between RCFTs and topological field theories?

In finding this connection, it is necessary to choose a topological, gauge invariant action. A likely candidate, the Chern-Simons functional, had recently been studied in detail because of its importance in the Lagrangian formulation of the Wess-Zumino-Witten model discussed in part $I$ of this thesis. Finding a topological classical action, however, is not enough. In quantizing a classical action it is necessary to prescribe a regularization scheme, which involves choosing a Riemannian metric for the space-time, thus possibly ruining the general covariance of the theory. It is necessary to show that the regularized quantities, that is, the relevar.t determinants, are in fact topological invariants. In the weak-field limit of the quantum theory, we can concentrate on the path integral about the classical field equation solutions, or in this case the flat gauge connections. In this limit, with a natural choice of regularization scheme, Schwarz showed that the path integral gives rise to the Ray-Singer analytic torsion of the flat connection being expanded about $[48,59]$. The Ray-Singer torsion of a flat gauge connection is a known topological invariant, and thus in the weak field 
limit the existence of a true topological quantum field theory is demonstrated.

At finite coupling, the use of quantum field theory techniques to show that topological invariants exist is more subtle. Hence a large effort from both the mathematics and physics communities has been made in putting this process on firmer ground. Aside from bringing together researchers from these often distant branches, a great development has been seen in the fields of integrable models and quantum groups, as well as axiomatic topological quantum field theory. This work has culminated in a direct connection between the existence of a certain class of quantum groups, the "ribbon Hopf algebras," and the knot invariants found by Witten. Thus the ambiguities of the quantum field theory have been circumvented, or more precisely, they have been axiomatized in the language of Hopf algebras.

The connection between the Chern-Simons quantum theory and RCFTs appears also in the structure of the Chern-Simons Hilbert space without sources $[18,19,28,41,42]$ (that is, without knots present.) In the following section of this thesis, we demonstrate that the Hilbert space of Chern-Simons theory with space-time manifold $\Sigma \otimes \mathbf{R}$ ( $\Sigma$ being the two-torus and $\mathbf{R}$ the real line) provides the fusion rules of the corresponding Wess-Zumino-Witten model, as well as an efficient method of explicitly calculating the modular transformations on the primary fields. The effect of monopole background charges is also considered in detail. The mathematical structure is surprisingly intricate and allows a clear geometric description.

\section{The Chern-Simons Action Functional}

Let $\mathrm{M}$ be a 3-manifold. The gauge field $A_{\mu}$ is a one-form on $\mathrm{M}$ taking values in $\mathbf{g}$, the Lie algebra of some semi-simple group $\mathrm{G}$. The Chern-Simons action functional is given by,

$$
\begin{aligned}
& A_{C S}=\frac{k}{4 \pi} \int_{M} \operatorname{Tr}\left(A \wedge d A+\frac{2}{3} A \wedge A \wedge A\right) \\
& =\frac{k}{4 \pi} \int_{M} d^{3} x \varepsilon^{\mu \nu \rho} \operatorname{Tr}\left(A_{\mu} \partial_{\nu} A_{\rho}+\frac{2}{3} A_{\mu} A_{\nu} A_{\rho}\right) .
\end{aligned}
$$

The Chern-Simons functional has a number of important properties. First, it is independent of the space-time metric. This will ensure that observable quantities will be generally covariant. A second property concerns its gauge 
invariance. Because the homotopy group $\pi_{3}$ of a simple Lie group $G$ satisfies: $\pi_{3}(G) \simeq \mathrm{Z}$, the set of gauge transformations is a disconnected set where each component can be labelled by an integer $m$, called the winding number. The Chern-Simons functional is invariant under gauge transformations connected to the identity (winding number zero) and changes by,

$$
A_{C S} \rightarrow A_{C S}+\text { constant } \cdot m
$$

under a general gauge transformation. The constant depends linearly on the normalization of the trace and on the overall constant $k$. We will normalize the trace so that the constant is given by $2 \pi \cdot k$. When computing the path integral, the contribution $\exp \left(i \cdot A_{C S}\right)$ will be invariant under a general gauge transformation if and only if $k \in \mathrm{Z}$, thus providing an argument for the quantization of $k$.

The Euler-Lagrange equations of motion give,

$$
\varepsilon^{\mu \nu \rho} F_{\nu \rho}=0
$$

where $F_{\nu \rho}$ is the field strength tensor. In other words, the field equations are satisfied if and only if the field strength vanishes.

\section{Quantization of the Chern-Simons Action}

We study the quantization of the Chern-Simons action functional restricted to the case where the 3-manifold, $M$, is diffeomorphic to the product of a 2torus, $\Sigma$, and the real line. With appropriately quantized $k$, the action is gauge invariant, and we choose the axial (i.e. physical) gauge $A_{t}=0$ (with $\mathrm{t}$ defining the coordinate along the real line in $\mathrm{M}$ ). After integrating by parts the action reduces to,

$$
I_{C S}=\frac{k}{2 \pi} \int_{M} d^{3} x \operatorname{Tr}\left(A_{1} \partial_{t} A_{2}\right)
$$

with the constraint (the equation of motion derived from varying $A_{t}$,)

$$
F_{12}=0=\partial_{1} A_{2}-\partial_{2} A_{1}+\left[A_{1}, A_{2}\right]
$$

Instead of first quantizing the system and then imposing the constraints as a projection on the Hilbert space, we will first determine classically the space of 
solutions to the constraint eq.(115) and quantize this simpler (finite dimensional) space.

The constraint $F_{\mu \nu}=0$ requires that the connection be flat. The space of flat connections on a Riemann surface $\Sigma$, modulo gauge transformations, is isomorphic to the space of maps,

$$
\phi: \pi_{1}(\Sigma) \rightarrow G
$$

modulo a global gauge transformation at the base point. Here $\pi_{1}(\Sigma)$ is the fundamental group of the manifold $\Sigma$. In our case $\Sigma$ is the torus, and we can present $\pi_{1}(\Sigma)$ as,

$$
\pi_{1}(\Sigma)=\left\{A, B \mid A B A^{-1} B^{-1}=1\right\}
$$

We represent the generators $A$ and $B$ as the holonomies of the gauge field $A_{\mu}$ about the two non-contractible loops $C_{1}$ and $C_{2}$ (see Figure 1) of the torus,

$$
\begin{aligned}
& A=P \exp \int_{C_{1}} A_{\mu} d x^{\mu}, \\
& B=P \exp \int_{C_{2}} A_{\mu} d x^{\mu} .
\end{aligned}
$$

In other words, the resulting classical phase space is given by the arbitrary embedding of $A$ and $B$ into the group $\mathrm{G}$, subject to the constraint $A B=B A$ and the global gauge transformation at the base point of $C_{1}$ and $C_{2}$. Since $A$ and $B$ commute, we can use this global gauge transformation to put them both in the maximal torus of $\mathrm{G}$. Given that any two flat gauge configurations with the same holonomies are equivalent up to gauge transformations, one can conveniently choose gauge field representations of a given class (or holonomy) to be constant over the torus. Also, since $A$ and $B$ are in the maximal torus of $\mathrm{G}$ one can require that the constant gauge field $A_{\mu}$ take values in the Cartan subalgebra of $g$. Thus, the path-ordered exponentials reduce to ordinary exponentials, and so,

$$
\begin{aligned}
& A=\exp \int_{C_{1}} A_{\mu} d x^{\mu}=e^{i a(t)} \\
& B=\exp \int_{C_{2}} A_{\mu} d x^{\mu}=e^{i b(t)}
\end{aligned}
$$

implicitly defining $a(t)$ and $b(t)$ as functions of the "time" variable taking values in the Cartan subalgebra of $\mathbf{g}$.

Substituting these identifications into the functional eq.(111), and letting the coordinate $x_{1}$ define the position on the torus along $C_{1}, x_{2}$ the position along 
$C_{2}$, we perform the integral over $\Sigma$. We have overlooked a possible change in the measure associated with the Jacobian arising from the change of variables $A_{\mu}$ to $A, B$. This is discussed in ref.[3,21, 22, 28, 40,41, 42, 59], where it is shown that the Jacobian simply shifts the coupling $k$ to $k+c$ where $c$ is the dual Coxeter number of the Lie algebra (in our normalization we have $c=\mathrm{N}$ for $\mathbf{g}=$ $\mathrm{SU}(\mathrm{N})$.) With this shift we have,

$$
I_{C S}=-\frac{k+c}{2 \pi} \int d t \operatorname{Tr}\left(a(t) \partial_{t} b(t)\right)
$$

At this point, we must choose a basis for the Cartan subalgebra of $g$. The classical observables $A$ and $B$ are periodic under certain shifts in $a(t)$ and $b(t)$. We would like to choose a basis which gives a primitive cell with respect to this periodicity. To ensure this, we choose the simple roots as our basis,

$$
a(t)=a_{i}(t) \nu^{i}, \quad b(t)=b_{i}(t) \nu^{i} .
$$

The $\nu^{i}$ are the simple roots. Once we have chosen a basis, we can perform the trace, where we define,

$$
\operatorname{Tr}\left(\nu^{i} \nu^{j}\right)=C^{i j}
$$

If $\mathrm{g}$ is simply-laced we can normalize the trace so that $C^{i j}$ is equal to the Cartan matrix. If $\mathbf{g}$ is not simply-laced, the above trace is not proportional to the Cartan matrix. For these algebras, $C^{i j}$ will be defined by eq.(122) where the normalization is determined by requiring that $\operatorname{Tr}\left(\nu^{1} \nu^{1}\right)=2$, and $\nu^{1}$ is a longer root. As an example of the simply-laced case, consider $\mathrm{G}=\mathrm{SU}(3)$. In the fundamental representation we have,

$$
\begin{gathered}
\nu^{1}=\left(\begin{array}{ccc}
1 & 0 & 0 \\
0 & -1 & 0 \\
0 & 0 & 0
\end{array}\right)=\tau^{3}, \\
\nu^{2}=\left(\begin{array}{ccc}
0 & 0 & 0 \\
0 & 1 & 0 \\
0 & 0 & -1
\end{array}\right)=-\frac{1}{2} \tau^{3}+\frac{\sqrt{3}}{2} \tau^{8} .
\end{gathered}
$$

And we see that it is natural to scale the trace by a factor of 2 to get the Cartan matrix from eq.(122). Note that choosing the simple roots in a different 
representation would result only in a different scaling. Therefore, from this point on we will work with the effective action,

$$
I_{C S}=-\frac{k+c}{2 \pi} \int d t a_{i}(t) C^{i j} \partial_{t} b_{j}(t),
$$

where $C^{i j}$ is equal to the Cartan matrix for simply-laced $g$ and given by eq.(122) for non-simply-laced $g$ (with the normalization prescription discussed below eq.(122).)

The action given in eq.(124) is linear in time derivatives, and thus canonical quantization gives the following commutation relations:

$$
\begin{gathered}
{\left[a_{i}, b_{j}\right]=-\frac{2 \pi i}{k+c}\left(C^{-1}\right)_{i j},} \\
{\left[a_{i}, a_{j}\right]=0,} \\
{\left[b_{i}, b_{j}\right]=0 .}
\end{gathered}
$$

We define the quantum operators,

$$
A_{i}=e^{i a_{i}}, \quad B_{i}=e^{i b_{i}} .
$$

As an example, consider $\mathrm{G}=\mathrm{SU}(3)$ in the fundamental representation. (The simple roots were given above.) We have,

$$
A=\left(\begin{array}{ccc}
A_{1} & 0 & 0 \\
0 & A_{1}^{-1} A_{2} & 0 \\
0 & 0 & A_{2}^{-1}
\end{array}\right), \quad B=\left(\begin{array}{ccc}
B_{1} & 0 & 0 \\
0 & B_{1}^{-1} B_{2} & 0 \\
0 & 0 & B_{2}^{-1}
\end{array}\right) .
$$

The commutation relations of eq.(125) imply,

$$
\begin{aligned}
A_{i} B_{j} A_{i}^{-1} B_{j}^{-1} & =\exp \left(\frac{2 \pi i}{k+c}\left(C^{-1}\right)_{i j}\right), \\
A_{i} A_{j} & =A_{j} A_{i}, \\
B_{i} B_{j} & =B_{j} B_{i} .
\end{aligned}
$$

We realize these commutation relations on a finite dimensional Hilbert space. For simplicity of exposition, we will work out the details for $G=S U(N)$. For the simply-laced case, $C^{i j}$ is just the Cartan matrix. The column vectors of the inverse of the Cartan matrix define the fundamental weight vectors of the Lie algebra. We diagonalize the $A^{i}$ operators (they commute with each other) and define the eigenstates,

$$
A_{i} \psi_{\vec{u}}=\eta^{u_{i}} \psi_{\vec{u}}, \quad \text { where } \eta=\exp \left(\frac{2 \pi i}{N(k+N)}\right)
$$


In this basis, the $B_{i}$ operators act as raising operators along the fundamental weight vectors. We have,

$$
B_{i} \psi_{\vec{u}}=\psi_{\vec{u}+\vec{w}_{i}}
$$

where $w_{i}$ are the weight vectors. To see this explicitly, consider the example $\mathrm{N}=3, \mathrm{k}=1$. We have,

$$
\begin{gathered}
A_{1} \psi_{i j}=\eta^{i} \psi_{i j} \\
A_{2} \psi_{i j}=\eta^{j} \psi_{i j} \\
B_{1} \psi_{i j}=\psi_{i+2, j+1} \\
B_{2} \psi_{i j}=\psi_{i+1, j+2}, \\
\eta^{12}=1 .
\end{gathered}
$$

If we start at the state $i=0, j=0$, we can raise to any state such that $i+j=0$ $(\bmod 3)$. We call this restricted subspace the state space. Note that two states $\psi_{i j}$ and $\psi_{k l}$ are equivalent if and only if $i=k(\bmod 12)$ and $j=l(\bmod 12)$. In Figure 2 we have plotted these states. Note that the states $(3,0)$ and $(0,3)$ lie along the simple root vectors, and the states $(2,1)$ and $(1,2)$ lie along the primitive weight vectors.

Finally, we need to understand the residual gauge symmetry, Weyl invariance. It is clear how the Weyl reflections act on this state space (that is, by the usual action on the weight lattice). Vectors in this state space fall into various Weyl orbits. For our example, the result is shown in Figure $\hat{3}$. We see that for a simply-laced gauge group, the Hilbert space is,

$$
H=\frac{\Lambda_{W}}{(k+c) \Lambda_{R} \triangleright W},
$$

with $\Lambda_{W}$ the weight lattice, $\Lambda_{R}$ the root lattice and $W$ the Weyl group. Also, $\triangleright$ implies the semi-direct product. Note that in the case of $g$ non-simply-laced, this expression must be altered (see ref. [28] and the note below eq.(122).) The general result, consistent with the normalization discussed, is given by,

$$
H=\frac{\Lambda_{W}}{2(k+c) \Lambda_{r} \triangleright W},
$$

$\Lambda_{r}$ is the dual-root lattice, defined by the basis vectors $\alpha_{i} /\left|\alpha_{i}\right|^{2}, \alpha_{i}$ being the simple roots. 


\section{Modular Tranformations}

In the variables defined in Section 13, the modular transformations act in the following way,

$$
T:(a, b) \rightarrow(a, b-a), \quad S:(a, b) \rightarrow(b,-a) .
$$

We want to show that these transformations act on the operators $A_{i}$ and $B_{i}$ in a well defined, representation independent way. We start with the $T$ transformation. For the purpose of exposition consider the case $\mathrm{SU}(3)$ in the fundamental representation. Using eq.(125) we have,

$$
T(B)=e^{i(b-a)}=e^{\frac{1}{2}[a, b]} e^{i b} e^{-i a}=R B A^{-1},
$$

$R, A$, and $B$ are diagonal matrices in the Lie algebra space. For a general representation $R$ will contain different phases along the diagonal. In the fundamental representation, eq.(123) and eq.(127), we have,

$$
\begin{gathered}
a=a_{i} \nu^{i}=\left(\begin{array}{ccc}
a_{1} & 0 & 0 \\
0 & -a_{1}+a_{2} & 0 \\
0 & 0 & -a_{2}
\end{array}\right), \\
b=b_{i} \nu^{i}=\left(\begin{array}{ccc}
b_{1} & 0 & 0 \\
0 & -b_{1}+b_{2} & 0 \\
0 & 0 & -b_{2}
\end{array}\right), \\
R=\eta^{-1} I .
\end{gathered}
$$

The transformation eq.(135) can be realized with the operators $A_{i}$ and $B_{i}$ in the following way (see eq.(128) and eq.(129),)

$$
\begin{gathered}
T\left(A_{i}\right)=A_{i}, \\
T\left(B_{i}\right)=\eta^{-1} B_{i} A_{i}^{-1} .
\end{gathered}
$$

In Section 17 it is shown that the transformation given in eq.(138) is consistent with eq.(136) for any representation. It is also shown how this argument generalizes to arbitrary $\mathrm{g}$.

The $\mathrm{S}$ transformation is simpler, and it is easy to verify that the following transformations are consistent with eq.(135):

$$
\begin{gathered}
S\left(A_{i}\right)=B_{i}, \\
S\left(B_{i}\right)=A_{i}^{-1} .
\end{gathered}
$$


At this point, we need to realize these iransformations on the state space. For the purpose of exposition we restrict to the case $G=S U(N)$. It is not difficult to verify that these relations are satisfied by the identifications,

$$
\begin{gathered}
T_{\vec{u}, \vec{v}}=\delta_{\vec{u}, \vec{v}} \eta^{t}(\vec{u}), \text { with } t(\vec{u})=\frac{1}{2 N} \vec{u}^{T} A \vec{u}, \\
S_{\vec{u}, \vec{v}}=\frac{1}{\sqrt{N(k+N)^{2}}} \eta^{s}(\vec{u}, \vec{v}), \text { with } s(\vec{u}, \vec{v})=\frac{1}{N} \vec{u}^{T} A \vec{v},
\end{gathered}
$$

where $A$ is the Cartan matrix of SU(N). The normalization factor in front of $S$ ensures that $S$ is hermitian. It can be easily shown that $S$ and $T$ commute with Weyl reflections in this state space as required by gauge invariance.

At this point, we consider the shift of the vacuum by $\rho$. ( $\rho$ is one-half the sum of the positive roots (see ref.[28])). We notice that for $\mathrm{SU}(3)$, the number of maximal Weyl orbits at a given level $k$ (an orbit is maximal if its order is equal to the order of the Weyl group) is equal to the number of primary fields in the corresponding conformal field theory. Also, if we shift the vacuum by $\rho$, we see that each maximal orbit has a representative state equal to the highest weigh: of one of the integral representations (see Figure 4). For each maximal Weyl orbit, define a state $\psi_{a}$ in the state space, which is an eigenstate of the Weyl reflections corresponding to simple roots, with eigenvalue -1 . We make the following conjecture: for a given semi-simple gauge group $\mathrm{G}$, the collection of states $\psi_{a}$ defined above is in one-to-one correspondence with the primary fields of the corresponding WZW model. Furthermore, when the modular transformations $S$ and $T$, defined above for the state space, are projected onto this collection of states, the resulting transformations $S^{\prime}, T^{\prime}$ are equal to those calculated by Kac [37] (see also ref.[38, 45]) for the corresponding WZW model. In Section 17 we prove equivalence of these $S$ matrices for the case $\mathrm{G}=\mathrm{SU}(\mathrm{N})$ at arbitrary level $k$.

\section{Fusion Rules and the Verlinde Conjecture}

The fusion rules of the WZW model can now be easily understood in terms of Chern-Simons theory. We define the folowing gauge invariant operator:

$$
\Theta_{R}=\operatorname{Tr}_{R}(B)
$$


The subscript " $R$ " labels a given representation of $\mathrm{g}$, defining the representation in which the trace is to be taken. The operators $B_{i}$ act as raising operators in the state space defined in Section 13. For all examples we have checked, the operator $\Theta_{R}$ consistently projects onto the "primary field" states $\psi_{a}$, and gives the correct fusion algebra. That is, let $P$ be a representation and $\psi_{P}$ be the state associated ith that representation. We find that,

$$
\Theta_{R} \cdot \psi_{P}=N_{R P}^{Q} \psi^{Q}
$$

where $N_{R P}^{Q}$ are the fusion coefficients. To see this explicitly, consider the case G $=\mathrm{SU}(3)$. Letting $\Omega$ be the fundamental representation we have,

$$
\Theta_{R}=\operatorname{Tr}_{R}(B)=B_{1}+B_{1}^{-1} E_{2}+i_{-2}^{-1}
$$

In Figure 5 we demonstrate how this operator is used to derive the fusion rule $3 \otimes 8=3 \oplus \overline{6}$ for $\mathrm{SU}(3)$ level 2 . Note the strong similarity between this technique and that of Walton [54].

To further motivate eq.(143) note that since the $B_{i}$ s commute among themselves (see eq.(128)) $\Theta_{R_{1}} \cdot \Theta_{R_{2}}=\Theta_{R_{2}} \cdot \Theta_{R_{1}}$. It is more difficult to show that $N_{R P}^{Q}=N_{P R}^{Q}$ directly from eq.(143), but the commutativity and associativity of the $N_{R P S}^{Q}$ indicates that they are likely candidates for the fusion coefficients. Further, computation of $N_{R P}^{Q}$ via eq.(143) in many particular cases reproduces the known results [31]. We see that the fusion algebra is generated by Weylinvariant combinations of the operators $B_{i}$. In Section 2, it was shown that the modular transformations $S$ maps $B$ into $A^{-1}$ and that, on the state space $\mathrm{A}$ is diagonal. Thus $S$ diagonalizes $B$, and subsequently $S$ diagonalizes the fusion algebra. This is the celebrated Verline conjecture [51, 52].

\section{Background Gauge Fields and Monopoles}

Having developed the quantization of Chern-Simons theory to the point where the structure of the underlying conformal field theory emerges clearly and explicitly, we now generalize our construction to include classical (i.e., background) field configurations. The notion of coupling the degrees of freedom of a conformal model to background gauge fields is an old one $[1,2,8,35,39,50]$. In this 
section, we study Chern-Simons theory with background gauge fields, particularly focusing on the twisted sectors and the modular properties of these sectors in the presence of a monopole.

To begin with, again consider taking the gauge $A_{t}=0$ and satisfying the flatness condition $F_{12}=0$ on a torus with a point removed, $\Sigma$. We do not consider the holonomy around this point removed to be an additional quantum degree of freedom but instead specify the holonolmy about the hole entirely in terms of the classical part of the gauge field. By the monopole quantization condition we learn that the moduli space of flat connections modulo gauge transformations on $\Sigma$ is again 2R-dimensional ( $R$ is the rank of $G$ ). An excellent review of monopoles is ref.[24]. As before, a nonsingular gauge transformation may be used to put the gauge field entirely into the Cartan subalgebra. We then have the following decomposition:

$$
A_{\mu}=A_{\mu}^{(c)}+A_{\mu}^{(q)}
$$

Here $A_{\mu}^{(q)}$ is the quantum fluctuation about $A_{\mu}^{(c)}$ which may have both monopole and nontrivial holonomy about the canonical cycles of the torus (we imagine the monopoles as having all their flux concentrated at the point removed.) Since $A_{\mu}^{(c)}$ is a background field, it is time-independent and so, putting the decomposition eq.(145) into the action of eq.(111), we find the Poisson bracket (and thus the commutator) for $A_{\mu}^{(q)}$ is as before (eq.(125)).

The classical holonomy pieces "twist" the theory whereas the monopole pieces of $A_{\mu}^{(c)}$ simply changes the modular properties of the theory. We discuss these points in turn below.

As before, quantization consists of representing the operators $\tilde{A}=P \exp \int A$ as unitary operators on a vector space. Using the full $A_{\mu}$ of eq.(145) we see that the classical holonomy pieces of $A_{\mu}^{(c)}$ correspond to choices of boundary conditions for the vectors in the Hilbert space if viewed in terms of the operator $A=P \exp \int A^{(q)}$ :

$$
\tilde{A}_{i} \psi_{0}=\psi_{0} \Rightarrow A_{i} \psi_{0}=\gamma_{i} \psi_{0}, \text { with } \gamma_{i} \in U(1)
$$

Here the $\tilde{A}_{i}$ s are, as described before, components of the full $P \exp \int A$ and thus correspond to transport of the $\psi \mathrm{s}$ under $A_{\mu}$. Along the chosen cycle (here $C_{1}$, in a two-dimensional sense, is along the "time" direction), the individual 
$A^{\prime}$ s c correspond to components of $g(0) g^{-1}(2 \pi)$, and so requiring $Z_{N}$ boundary conditions for $\mathrm{g}$ is implemented by the $\gamma_{i}$ s being nontrivial phases. Following ref.[33], first implement the twisting along the $C_{1}$ cycle and then use modular transformations to build up the remaining shifted sectors. One may furthermore compute the fusion rules by following the procedure described in Section 15.

It was shown in ref.[8] that the modular properties of the theory on the torus depend on the topological type of the gauge field $A_{\mu}^{(c)}$. One way to understand this result heuristically is as follows: instead of concentrating the flux in a single point, imagine spreading it out evenly over the entire torus. Then $S$ simply interchanges the cycles whereas $T$ actually combines the cycles, as seen in Figure 6. The new cycle so generated $\left(C_{2}^{\prime}\right)$ is homotopically equivalent to $C_{1}^{-1} C_{2}$ but the path ordered exponential of $A_{\mu}$ along these cycles differ due to the fact that the triangle bounding them contains $1 / 2$ the flux of the monopole. This is the simple explanation of a more rigorous calculation in which one considers arbitrary flux distributions. Thus, in the presence of a monopole background of charge $n$, the $T$ matrix of eq.(138) is modified,

$$
T_{n}^{\prime}\left(A_{i}\right)=A_{i} \quad T_{n}\left(B_{j}\right)=s_{j, n} r_{j} B_{j} A_{j}^{-1}
$$

where $s_{j, n}$ is an additional phase that is a solution to eq.(170 of Section 17 and all other variables are as before (compare with eq.(138) of Section 14.) In Section 17 it is shown that for $\mathrm{SU}(\mathrm{N}) / Z_{N}, s_{j, n}=\alpha^{j n}$ where $\alpha^{N}=1$. As before, in the sector without a monopole $(n=0)$, the $T_{n}(n \neq 0)$ matrix may be found by studying eq.(147) on the untwisted sector. This matrix is then also used to study the modular properties of the twisted sectors.

Below is a brief summary of the effect of including nontrivial background gauge fields in Chern-Simons quantum mechanics on the torus:

1. Fusion Algebra of Twisted Sector. States in the twisted sector of eq.(146) and the untwisted sector may be fused as described in Section 15. One finds that the twisted representations behave as phased relabellings of the untwisted representations. The familiar example of $S U(2)_{2} / Z_{2}$ is given below in Figure 7.

2. Shifted Sectors. Shifted sectors are found in the usual fashion [33] by applying modular transformations to the untwisted and twisted sectors. We 
thus generate a modular covariant set of sectors. This may or may not lead to an irreducible representation of the modular group. For example, in $S U(2)_{k} / Z_{2}$, letting $(+,+)$ represent the untwisted sector and $(-,+)$ represent the twisted sector, one finds using the above $S$ and $T$ :

$$
\begin{aligned}
& T:(+,+) \text { invariant },(-,+) \rightleftharpoons(-,-), \\
& S:(+,+) \text { invariant },(-,+) \rightleftharpoons(+,-) .
\end{aligned}
$$

3. Monopoles and the Modular Properties of the Twisted Sector. Including monopole backgrounds in the theory causes $\mathrm{T}$ to change. With this modified $\mathrm{T}$ matrix, one may find modular properties of the theory in higher genus. Continuing with our example $S U(2)_{k} / Z_{2}$ and using the procedure described above, we find that $T_{1}$ given by eq.(147) yields,

$$
T_{1}:(+,+) \rightleftharpoons(+,-),(-,+) \text { invariant, }
$$

and thus we see that, combined with eq.(148), the representation of the modular group on the four "spin connections" is irreducible.

4. Monopoles and Projective Representations of the Modular Group. Although the $T_{n}$ of eq.(147) do satisfy $\left(S T_{n}\right)^{3}=1$ as abstract group elements on the $A_{i} \mathrm{~s}$ and $B_{i} \mathrm{~s}$, one discovers that as matrices on the Hilbert space constructed above $\left(S T_{n}\right)^{3}=\delta(k, n) \cdot I$ where $\delta(k, n)$ is a phase that depends on the level and the monopole charge. Note that $\delta(k, 0)=1 \forall k$ but that for $n \neq 0$ this phase is not one and cannot be removed by unitary transformations. (Of course, trying to redefine $T_{n}$ by a phase would mean that as abstract generators $S$ and $T_{n}$ would no longer satisfy the defining relations of the modular group.) For the example above one finds,

$$
\text { For } \mathrm{g}=S U(2)_{k} / Z_{2}, \delta(k, 1)=-(-i)^{k} .
$$

Therefore including monopole backgrounds compels one to consider only projective representations of the modular group. This is expected for the representation of the modular group at higher genus.

\section{Three Necessary Calculations}

\section{1) Representation Dependence of Modular Transformation}


Recall that the modular transformation $\mathrm{T}$ maps,

$$
T(B)=e^{\frac{1}{2}[a, b]} B A^{-1} .
$$

This is a representation dependent statement, since $a$ and $b$, as well as $A$ and $B$ are expanded in some representation (see eq.(120) and eq.(122).) We want to show that eq.(151) can be projected consistently onto a modular transformation of the operators $B_{i}$. That is, we ask that there exist a consistent mapping,

$$
T\left(B_{i}\right)=r_{i} B_{i} A_{i}^{-1}
$$

for some set of phases $R_{i}$ and such that eq.(151) will hold for any representation. Let $P$ be such a representation and let $w$ be the $j$-th weight in this representation (in some arbitrary ordering). This weight can be expanded in the weight basis as,

$$
w=\sum_{i} n_{i} a_{i}
$$

with $A_{i}$ the primitive weight vectors and $n_{i}$ a set of integers. Then the corresponding diagonal element of $\nu^{i}$ in the expansion of eq.(121) is given by the coefficient $n_{i}$. The consistency check thus reduces to a check for any given weight in the weight lattice. Letting $(T(B))_{j}$ correspond to the $j$-th diagonal element of $T(B)$, we require,

$$
(T(B))_{j}=\prod_{i}\left(T\left(B_{i}\right)\right)^{n_{i}}
$$

Inserting eq.(151) and eq.(152), we have,

$$
\left(e^{\frac{1}{2}[a, b]}\right)_{j}(B)_{j}\left(A^{-1}\right)_{j}=\prod_{i}\left(r_{i} B_{i} A_{i}^{-1}\right)^{n_{i}} .
$$

Which can be further expanded to,

$$
e^{\frac{1}{2}\left[n_{i} a_{i} n_{i} b_{i}\right]} \prod_{i} B_{i}^{n_{i}} \prod_{i} A_{i}^{-n_{i}}=\prod_{i} r_{i}^{n_{i}} \prod_{i}\left(B_{i} A_{i}^{-1}\right)^{n_{i}} .
$$

On the right side, we can commute the $B_{i}$ factors through to the left by using the commutation relations of eq.(128). Also, the commutator on the left side can be computed using eq.(125). When this is done, the operator content is identical on both sides of the equation and so we need compare only the resulting phases. Equality demands,

$$
\beta^{-\frac{1}{2} \sum_{i, j} n_{i}\left(C^{-1}\right)_{i j} n_{j}}=\prod_{i} r_{i}^{n_{i}} \prod_{i} \beta^{-\left(C^{-1}\right)_{i i} \frac{n_{i}\left(n_{i}-1\right)}{2}} \prod_{i<j} \beta^{-\left(C^{-1}\right)_{i j} n_{i} n_{j}}
$$


where we have defined,

$$
\beta=\exp \left(\frac{2 \pi i}{k+c}\right)
$$

We can satisfy eq.(157) for arbitrary integers $n_{i}$, and therefore arbitrary representations, simply by letting,

$$
r_{i}=\beta^{-\frac{1}{2}\left(C^{-1}\right)_{i i}}
$$

2) Equivalence of Modular Transformation S from Chern-Simons Theory with Kac Formula for $S U(N)$ Models

We prove that the formalism outlined in Sections 13 and 14 correctly generates the modular tansformation $S$ for the case $G=S U(N)$, level k. We start by defining a map $\phi$ from the root-space of $g$ into an $\mathrm{N}$-dimensional vector space V.

$$
\phi: \alpha_{i} \rightarrow \eta_{i}-\eta_{i+1}
$$

where the $\alpha_{i}$ s are the N-1 simple roots, a faithful basis of the root-space. In V, the action of the Weyl group is simply permutation of coefficients of the vector. To see this simply consider the action of the Weyl reflections corresponding to simple roots acting on this basis,

$$
W_{i}\left(\alpha_{j}\right)=\alpha_{j}-A_{j i} \alpha_{i}=\left\{\begin{array}{cc}
-\alpha_{j} ; & i=j \\
\alpha_{i}+\alpha_{j} ; & |i-j|=1 \\
\alpha_{j} ; & |i-j|>1
\end{array} .\right.
$$

In $\mathrm{V}, W_{i}$ acts by permuting the $i^{\text {th }}$ and $i+1^{\text {st }}$ coefficients. Consistency is easily checked. Let $\Phi$ be the realization of $\phi$ in the simple-root basis. Define the matrix $K$,

$$
K=\Phi A^{-1},
$$

where $A$ is the Cartan matrix for $\mathrm{SU}(\mathrm{N})$. At this point, we note that the matrix $K$ is exactly the mapping appearing in the formula of Kac [37] for the $S$ matrix of $\mathrm{SU}(\mathrm{N})$, taking the Dynkin coefficients $\left(a_{1}, \ldots, a_{N-1}\right)$ into the vector $\left(\phi_{1}, \ldots, \phi_{N-1}\right)$ with the appropriate shift $\rho=(1, \ldots, 1)$.

Our prescription for $\mathrm{S}$ outlined in Sections 13 and 14 gives,

$$
S_{u, v}=\sum_{u}(-1)^{\operatorname{sign}(w)} \eta^{u A w(v)) / N}
$$


where $\eta^{N(k+N)}=1$; w is summed over the Weyl group; and $\operatorname{sign}(\mathrm{w})$ is $+1(-$ 1) if $w$ is expressible as an even (odd) product of simple-root Weyl reflections. Note also that $\mathrm{u}, \mathrm{v}$ are vectors in the root basis, not in the weight basis. After changing basis, employing eq.(162), and noting that $w$ acts by a permutation matrix in the correct basis (defined above), we can write,

$$
S_{a, b}=\sum_{w}(-1)^{\operatorname{sign}(w)} \exp \left\{\frac{2 \pi i}{N+K} \phi_{a}^{T}\left(P_{w}\right) \phi_{b}\right\} .
$$

This expression involves a sum over permutations. With the correct signs observed, this is simply a determinant. We have,

$$
S_{a, b}=\operatorname{det}(M) \text {, }
$$

where $\mathrm{M}$ is given by;

$$
M(a, b)_{i j}=\exp \left\{\frac{2 \pi i}{N+k} \phi_{i}(a) \phi_{j}(b)\right\} .
$$

This is the Kac formula [36, 37].

\section{3) Representation Independent Computation of $T_{n}$}

We show that for the $T_{n}$ of eq.(147) which represents the action of the $\mathrm{T}$ transformation on the $A_{i}$ and $B_{i} \mathrm{~s}$ in the presence of a monopole charge $\mathrm{n}$, the $s_{i, n}$ are independent of the representation chosen for the monopole field.

Whatever representation one chooses for the monopole background, one knows that $T_{n}$ is still a diagonal matrix on the Hilbert space and that, as described in the text, $T_{n}$ can, at most, pick up an additional phase $s_{i, n}$. Furthermore, whatever representation one chooses for the monopole, the resulting $T_{n}$ matrix must be gauge invariant. We have found that in some cases the imposition of gauge invariance is also a sufficient condition to ascertain all the monopole contributions to the theory. In our construction we have locally fixed the gauge but, as described in the text, one must impose gauge invariance with repect to large gauge transformations, i.e., the Weyl transformations. Thus one requires,

$$
w^{-1} T_{n} w=T_{n} \quad \forall w \in W,
$$

where $\mathrm{W}$ is the Weyl group. Recall that, in any representation Pexp $\int \mathrm{A}$ will have components of the form $\prod_{i} A_{i}^{d_{i}}$ where the $d_{i}$ s are the dynkin indices of 
the particular representation and $A_{i} \mathrm{~s}$ are, for example, as in eq.(126). We now study the individual $A_{i} \mathrm{~s}$ (and $B_{i} \mathrm{~s}$ ) abstractly without reference to any particular representation and see for example how they transform under $T_{n}$ and W. In general, then,

$$
T_{n}\left(A_{i}\right)=A_{i} \quad T_{n}\left(B_{i}\right)=r_{i} B_{i} A_{i}^{-1},
$$

where the $r_{i}, 1 \leq i \leq \operatorname{rank}(G)$ are some phases. For the Weyl reflection about the plane orthogonal to the $l^{\text {th }}$ root we have,

$$
\begin{gathered}
w_{l}\left(A_{j}\right)=A_{j} \text { for } j \neq l \text { and } \\
w_{l}\left(A_{l}\right)=A_{l} \prod_{m} A_{m}^{-C_{l}^{m}}
\end{gathered}
$$

as well as a similar equation for the $B_{i}$ s under $w_{l}$. Note that the necessary condition $\left(w_{l}\right)^{2}=I$ follows from $C_{m}^{m}=2 \forall m$.

Now we simply use eq.(168) and eq.(169) in eq.(167) and ask what the list of acceptable $r_{j}$ s is. We find that gauge invariance of $T_{n}$ (eq.(167)) implies the following general condition on the $r_{j} \mathrm{~s}$,

$$
r_{l}^{2} \prod_{m \neq l} r_{m} \prod_{j}\left(\frac{\zeta_{l j}^{2}}{\zeta_{j j}}\right)^{\frac{1}{2} C_{l}^{j}} \prod_{i, j} \zeta_{i j}^{-\frac{1}{2} C_{l} C_{l}^{j}}=1 \forall l,
$$

where $\zeta_{i j}$ are phases defined by,

$$
A_{i} B_{j} A_{i}^{-1} B_{j}^{-1}=\zeta_{i j}
$$

For example, for $\mathrm{SU}(\mathrm{N})$ using eq.(157) as a starting point,

$$
r_{j}=s_{j, n} \beta^{-\frac{1}{2}\left(C^{-1}\right) j j},
$$

where the $s_{j, n}$ represent additional phases due to the presence of the monopole. Then, using eq.(172) in eq.(170) we find that all k-dependence cancels and one has,

$$
s_{l}^{-2} \prod_{m \neq l} s_{m}^{-C_{l}^{m}}=1 \quad \forall l,
$$

where we have suppressed the $\mathrm{n}$ index (same for all the $s_{j}$ s above.) For $\mathrm{SU}(\mathrm{N})$ eq.(173) readily admits the solution $S_{j, n}=\alpha^{j n}$ where $\alpha$ is a primitive $N^{\text {th }}$ root of unity. 
18 Figures

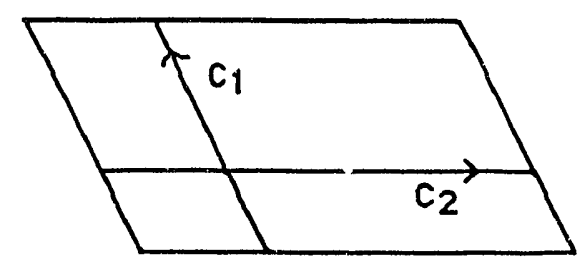

Figure 1

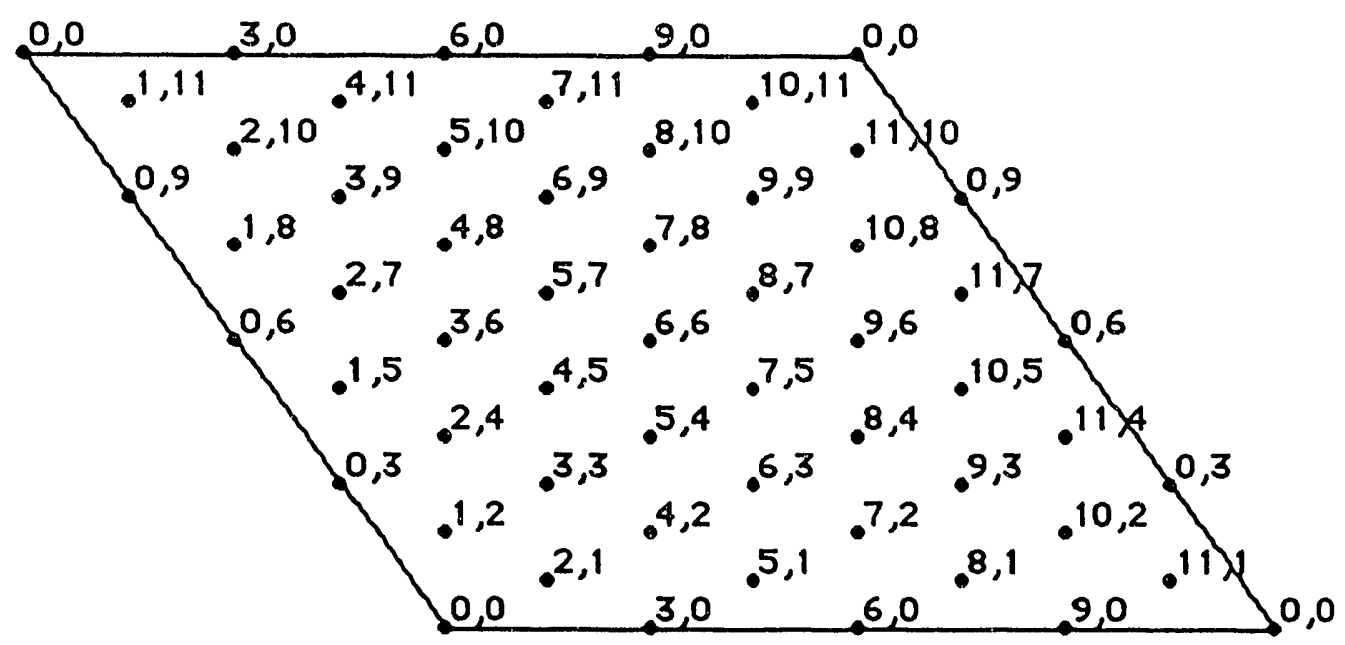

Figure 2

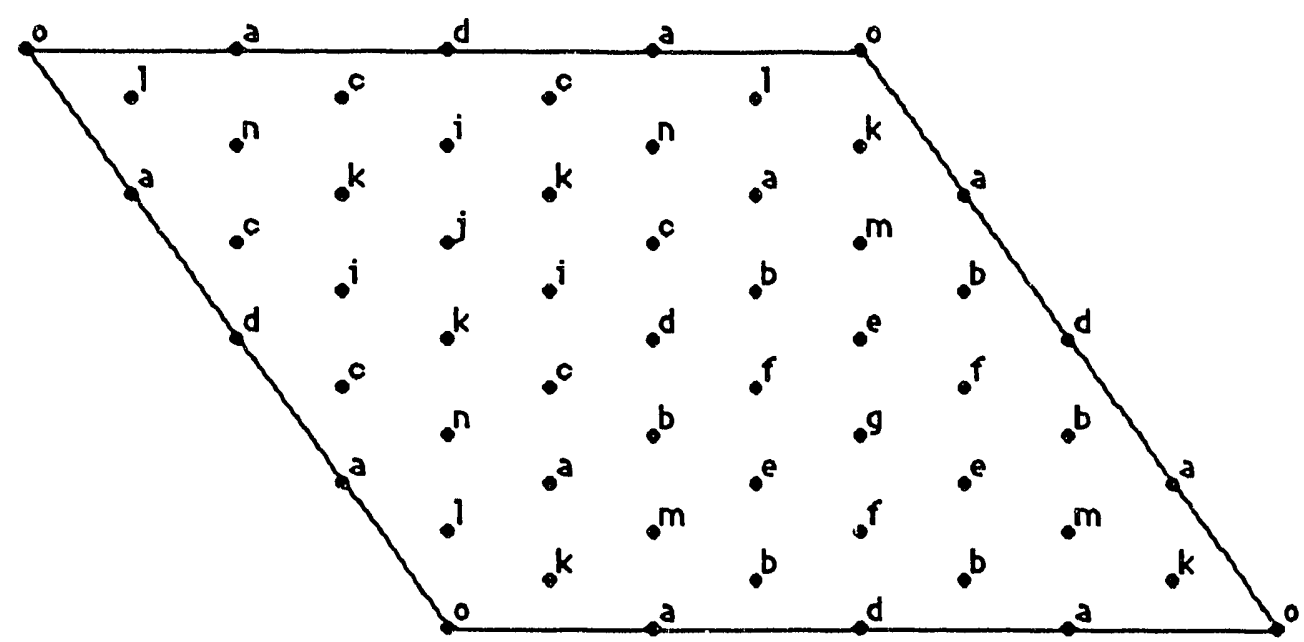

Figure 3 


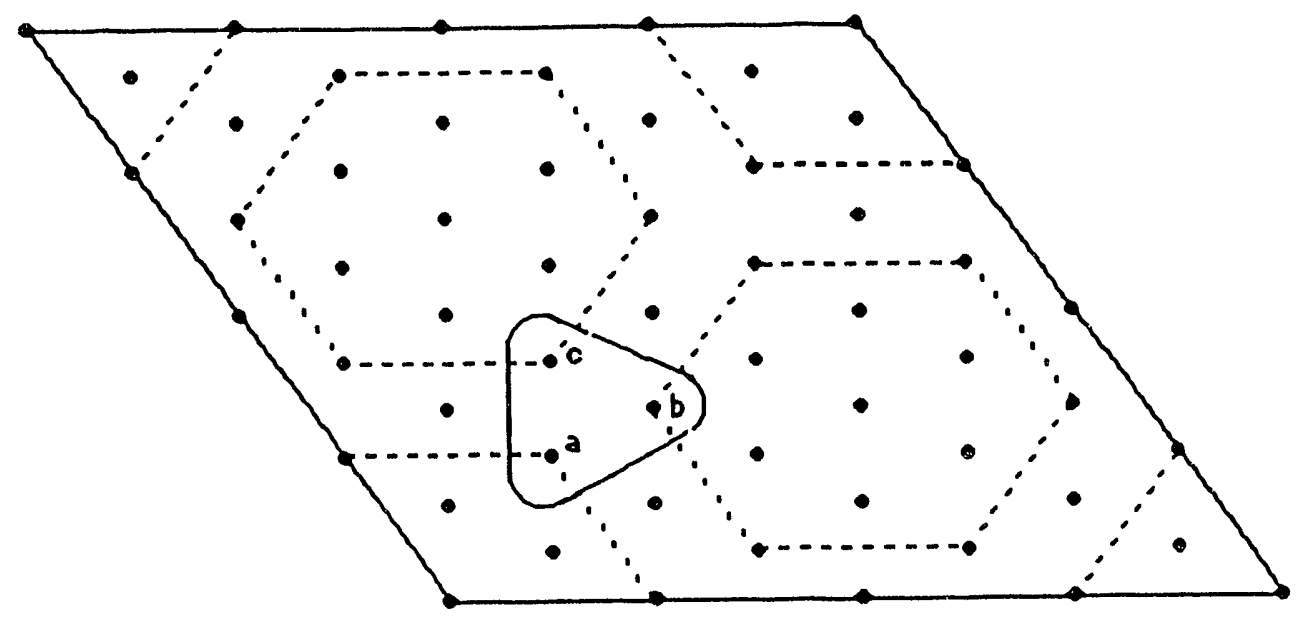

Figure 4

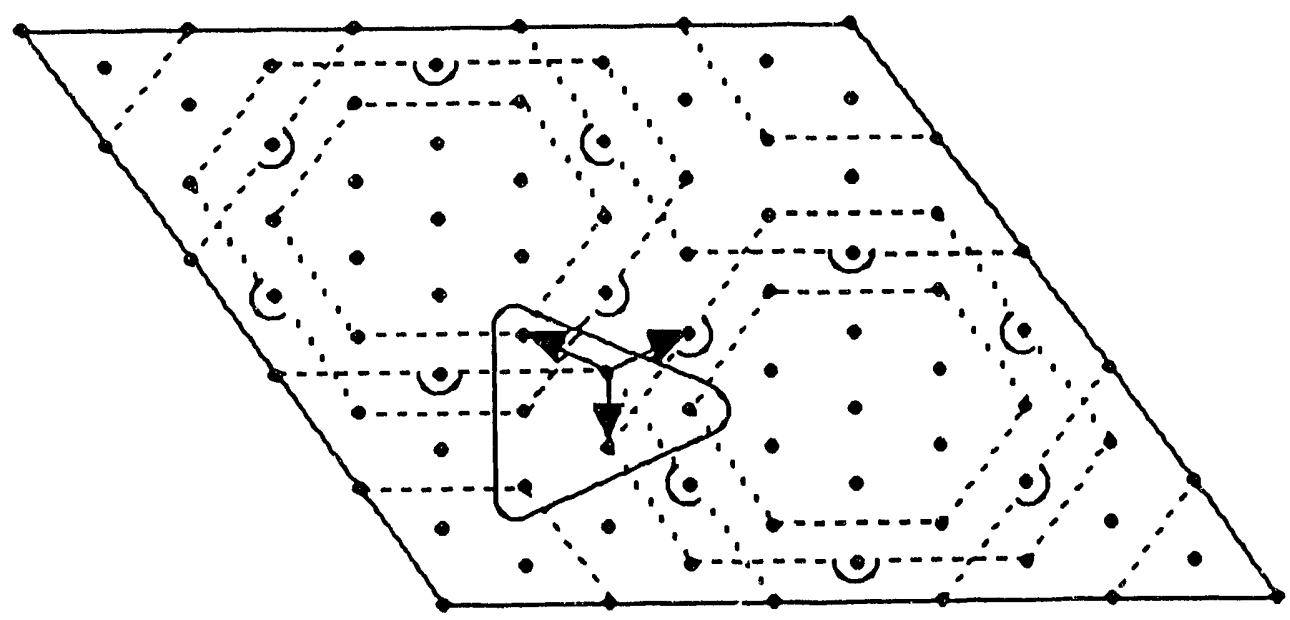

Figure 5 -- the fusion rule $3 \times \mathbf{8}=\overline{6}+3$ is demonstrated. Starting at each state in the orbit of the 8 representation, we operate with $\operatorname{Tr}(B)$ in the 3 representation. Taking into account relevant sign cancellations only the states of the $\overline{\mathbf{6}}$ and 3 orbits remain. 


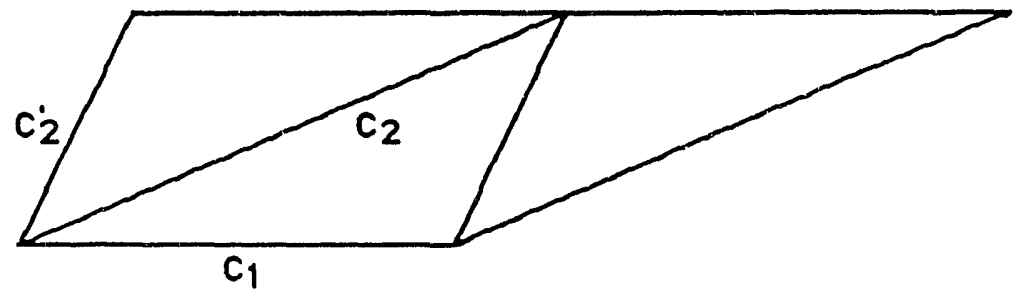

Figure 6

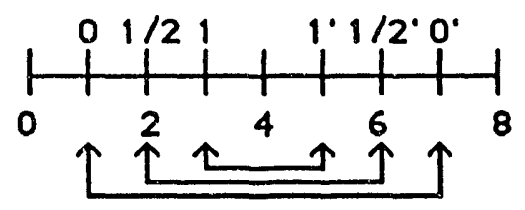

Fusion rules : $\quad 0 \times S=S$ for all $S$

$$
0^{\prime} \times S=S^{\prime} \text { for all } S \text { (note }\left(S^{\prime}\right)^{\circ}=S \text { ) }
$$

$$
\begin{array}{lll}
1 / 2 \times 1 / 2=0+1 & 1 / 2 \times 1 / 2^{\prime}=0^{\prime}+1^{\circ} & 1 / 2^{\circ} \times 1 / 2^{\circ}=0+1 \\
1 / 2 \times 1=1 / 2 & 1 / 2 \times 1^{\prime}=1 / 2^{\prime} & 1 / 2^{\prime} \times 1^{\prime}=1 / 2 \\
1 \times 1=0 & 1 / 2^{\circ} \times 1=1 / 2^{\prime} & 1 \times 1^{\prime}=0 \\
& 1 \times 1^{\circ}=0^{\circ} &
\end{array}
$$

Figure 7 -- $\ln G=S U(2)$ there is one twisted sector. The combined fusion rules are shown. These can be derived by defining the Verlinde operators of the primed fields as $\operatorname{Tr}(B)$, where the trace is taken in the spin 2,5/2 and 3 representations for states $1^{\prime}, 1 / 2$ and $0^{\prime}$ respectively. 


\section{References}

[1] A. Abouelsaood, C.G. Callan, C.R. Nappi, S.A. Yost, "Open Strings in Background Gauge Fields," Nucl. Phys. B280 (1987) 599.

[2] I. Affleck, J. Harvey, L. Palla, G. Semenoff, "The Chern-Simons Term Versus the Monopole," Nucl. Phys. B328 (1989) 575.

[3] L. Alvarez-Gaume, J.M.F. Labastida, A.V. Ramallo, "A Note on Perturbative Chern-Simons Theory," Nucl. Phys. B334 (1990) 103.

[4] F. Bais, P. Bouwknegt, M. Surridge, K. Schoutens, "Coset Construction for Extended Virasoro Algebras," Nucl. Phys. B304 (1988) 371.

[5] I. Bakas, E. Kiritsis, "Bosonic Realization of a Universal W-Algebra and $Z_{\infty}$ Parafermions," Nucl. Phys. B343 (1990) 185.

[6] I. Bakas, E. Kiritsis, "Grassmannian Coset Models and Unitary Representations of $W_{\infty}$," Berkeley preprint UCB-PTH-90/16 (1990).

[7] K. Bardakci, M.B. Halpern, "New Dual Quark Models," Phys. Rev. D. 3 (1971) 2493.

[8] K. Bardakci, M. Crescimanno, "Monopole Backgrounds on the World Sheet," Nucl. Phys. B313 (1989) 269.

[9] K. Bardakci, M. Crescimanno, E. Rabinovici, "Parafermions From Coset Models," Nucl. Phys. B344 (1990) 344.

[10] K. Bardakci, M. Crescimanno, S.A. Hotes, "Parafermions From nonAbelian Coset Models," Nucl Phys. B349 (1991) 439.

[11] K. Bardakci, M. Crescimanno, S.A. Hotes, "Classical W Algebras and NonAbelian Parafermions," Phys. Lett. 257B (1991) 313.

[12] K. Bardakci, "The Classical Parafermion Algebra, its Generalization and its Quantization," Berkeley preprint UCB-PTH-91/40 (1991).

[13] K. Bardakci, "Non-Abelian Paracurrents and Their Generalizations," Berkeley preprint UCB-PTH-92/02 (1992). 
[14] A. Belavin, A.M. Polyakov, A. Zamalodchikov, "Infinite Conformal Symmetry in Two-Dimensional Quantum Field Theory," Nucl. Phys. B241 (1984) 333.

[15] A. Bilal, "A Remark on the $\mathrm{N} \rightarrow \infty$ Limit of $W_{N}$-Algebras," Phys. Lett. 227B (1984) 406.

[16] A. Bilal, "Bosonization of $Z_{N}$ Parafermions and $s u(2)_{N}$ Kac-Moody Algebra," Phys. Lett. 226B (1989) 272.

[17] A. Bilal, "W Algebra Extended Conformal Theories, Cosets and Integrable Lattice Models," Cern preprint CERN-TH 5567/89 (1989).

[18] M. Bos, V.P. Nair, "U(1) Chern-Simons Theory and $c=1$ Conformal Blocks," Phys. Lett. 223B (1989) 61.

[19] M. Bos, V.P. Nair, "Coherent State Quantization of Chern-Simons Theory," Int. Journ. Mod. Phys. A5 (1990) 959.

[20] M. Caselle, G. Ponzano, F. Ravanini, "Towards a Classification of Fusion Rule Algebras in Rational Conformal Field Theories," Saclay preprint SPhT/91-174 (1990).

[21] W. Chen, G.W. Semenoff, Y. Wu, "Probing Topological Features In Perturbative Chern-Simons Gauge Theory," Mod. Phys. Lett. A5 (1990) 1833.

[22] W. Chen, G.W. Semenoff, Y. Wu, "Finite Renormalization of Chern-Simons Gauge Theory," Preprint 89-0956(UTAH) (1989).

[23] M. Crescimanno, S.A. Hotes, "Monopoles, Modular Invariance and ChernSimons Field Theory," Nucl. Phys. B372 (1992) 683.

[24] S. Coleman, "The Magnetic Monopole Fifty Years Later," Based on lectures given at the School of Sub-Nuclear Physics, "Ettore Majorana" (1981).

[25] S. Deser, R. Jackiw, S. Templeton, "Three Dimensional Massive Gauge Theories," Phys. Rev. Lett. 48 (1983) 975.

[26] S. Deser, R. Jackiw, S. Templeton, "Topologically Massive Gauge Theory," Ann. Phys. NY 140 (1984) 372. 
[27] V.G. Drinfeld, "Quasi-Hopf Algebras," Leningrad Math. J. 1 (1990) 1416.

[28] S. Elitzur, G. Moore, A. Schwimmer, N. Seiberg, "Remarks on the Canonical Quantization of the Chern-Simons-Witten Theory," Nucl. Phys. B326 (1989) 108.

[29] V.A. Fateev, A.B. Zamaludchikov, "Conformal Quantum Field Theory Models in Two Dimensions Having $Z_{3}$ Symmetry," Nucl. Phys. B280 (1987) 644.

[30] D. Friedan, Z. Qiu, S. Shenker, "Conformal Invariance, Unitarity, and Critical Exponents in Two Dimensions," Phys. Rev. Lett. 52 (1984) 1575.

[31] D. Gepner, E. Witten, "String Theory on Group Manifolds," Nucl. Phys. B278 (1986) 493.

[32] D. Gepner, "Field Identification in Coset Conformal Field Theories," Phys. Lett. 222B (1989) 207.

[33] P. Ginsparg, "Applied Conformal Field Theory," Les Houches (North Holland, Amsterdam) XLIX (1988).

[34] P. Goddard, A. Kent, D. Olive, "Virasoro Algebras and Coset Space Models," Phys. Lett. 152B (1985) 88.

[35] F.D.M. Haldane, E.H. Rezayi, "PeriodicLaughlin-Jastrow Wave Functions for the Fractional Quantized Hall Effect," Phys. Rev. B 3i (1985) 2529.

[36] V.G. Kac, D.H. Peterson, "Infinite-Dimensional Lie Algebras, Theta Functions and Modular Forms," Adv. in Math. 53 (1984) 125.

[37] V.G. Kac, M. Wakimoto, "Modular and Conformal Invariance Constraints in Representation Theory of Affine Algebras," Adv. in Math. 70 (1988) 156.

[38] E.J. Mlawer, S.G. Naculich, H.A. Riggs, H.J. Schnitzer, "Group- Level Duality of WZW Fusion Coefficients and Chern-Simons Link Observables," Nucl. Phys. B352 (1991) 863. 
[39] B.A. Ovrut, S. Thomas, "The Theory of Vortices and Monopoles on a Sphere," Phys. Rev. D 43 (1991) 1314.

[40] R.D. Pisarski, S. Rao, "Topologically Massive Chromodynamics in the Perturbative Fegime," Phys. Rev. D 32 (1985) 2081.

[41] A.M. Polyakov, P.B. Wiegmann, "Theory of Nonabelian Goldstone Bosons in Two Dimensions," Phys. Lett. 131B (1983) 121.

[42] A.M. Polyakov, P.B. Wiegmann, "Goldstone Fields in Two Dimensions With Multivalued Actions," Phys. Lett. 141B (1984) 223.

[43] A.P. Polychronakos, "Abelian Chern-Simons Theories in $(2+1)$ - Dimensions," Ann. Phys. 203 (1990) 231.

[44] A.P. Yolychronakos, "Abelian Chern-Simons Theories and Conformal Blocks," Preprint UFIFT-HEP-89-9 (1989).

[45] A.N. Redlich, H.J. Schnitzer, K. Tssokos, "Bose-Fermi Equivalence on the Two-dimensional Torus for Simply-laced Groups," Nucl. Phys. B289 (1987) 397.

[46] A.N. Schellekens, S. Yankielowicz, "Field Identification Fixed Points in the Coset Construction," Nucl. Phys. B334 (1990) 67.

[47] A.N. Schellekens, S. Yankielowicz, "Simple Currents, Modular Invariants and Fixed Points," Int. J. Mod. Phys. A5 (1990) 2903.

[48] A. Schwarz, "The Partition Function of Degenerate Quadratic Functionals and Ray-Sir.ger Invariants," Lett. Math. Phys. 2 (1978) 247.

[49] H. Sugawara, "A Field Theory of Currents," Phys. Rev. 170 (1968) 1659.

[50] E.T. Tomboulis, "Gauge Fields on the World Sheet: Strings with Gauge and Supersymmetry Breaking," Phys. Lett. 198B (1987) 165.

[51] E. Verlinde, "Fusion Rules and Modular T.ransformations in 2D Conformal Field Theory," Nucl. Phys. B300 (1988) 360.

[52] H. Verinde, E. Verlinde, "Conformail Field Theory and Geometric Quantization," Preprint PUPT 8C/1149 (1989). 
[53] M. Wakimoto, "Fock Representations of the Affine Algebra $A_{1}^{(1)}$," Comm. Ma.th, Phys. 104 (1986) 605.

[54] M.A. Walton, "Fusion Rules in Wess-Zumino-Witten Models," Nucl. Phys. B340 (1990) 777.

[55] G.M.T. Watts, "Determinant Formulae for Extended Algebras in Two Dimensional Conformal Field Theory," Nucl. Phys. B326 (1989) 648.

[56] G.M.T. Watts, "WB Algebra Representation Theory," Nucl. Phys. B339 (1990) 177.

[57] E. Witten, "Global Aspects of Current Algebra," Nucl. Phys. B223 (1983) 422.

[58] E. Witten, "Non-abelian Bosonoization in Two Dimensions," Comm. Math. Phys. 92 (1984) 455.

[59] E. Witten, "Quantum Field Theory and the Jones Polynomial," Commun. Math. Phys. 121 (1989) 351.

[60] A.B. Zamolodchikov, "Infinite Additional Symmetries in Two-Dimensional Conformal Quantum Field Theory," Theor. Math. Phys. 65 (1985) 1205.

[61] A.B. Zamolodchikov, V.A. Fateev, "Non-local (Parafermion) Currents in Two-Dimensional Conformal Quantum Field Theory and Self-Dual Critical Points in $Z_{N}$-Symmetric Statistical Systems," Sov. Phys. J.E.T.P. 62 (1985) 215. 

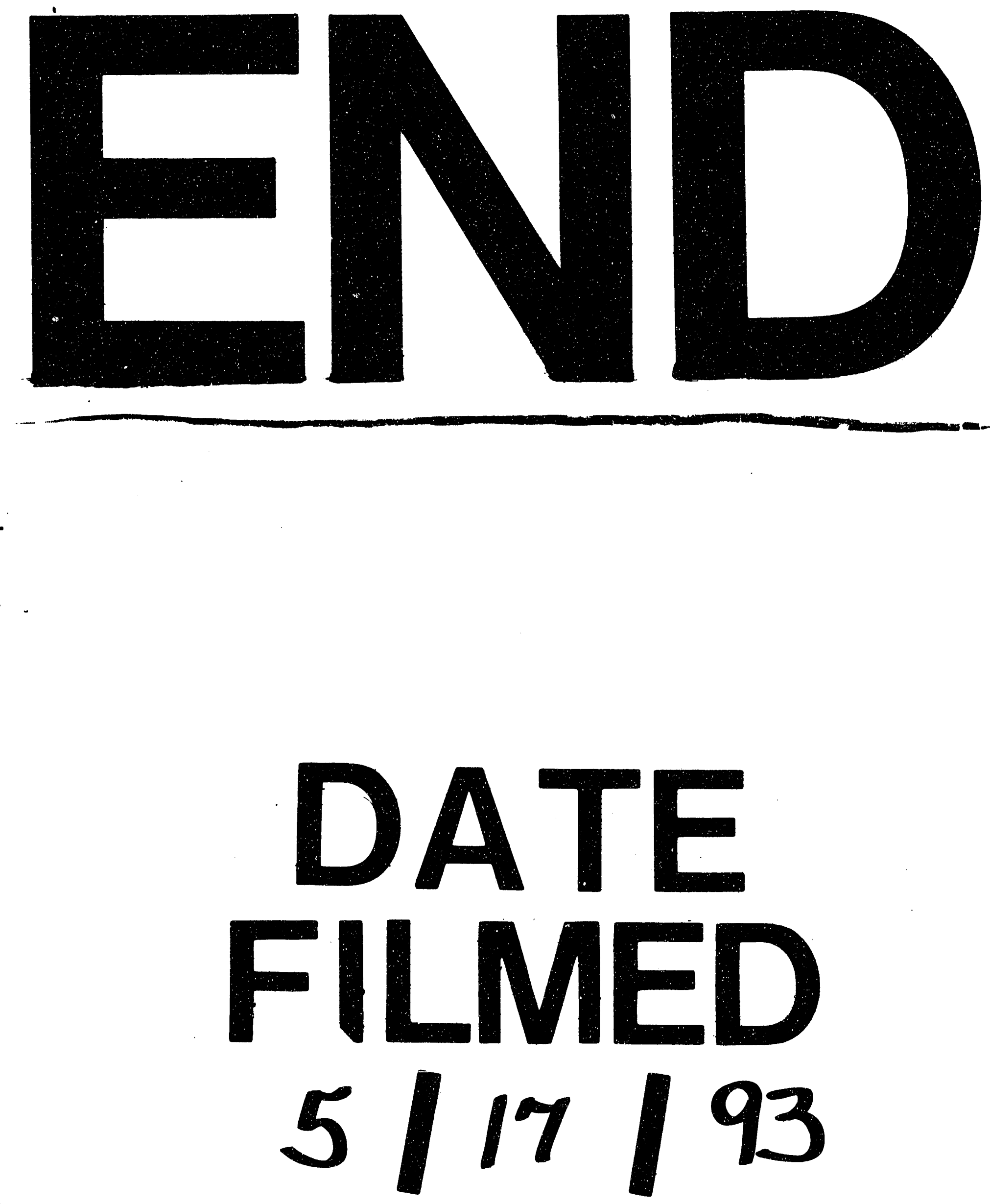
